\title{
Scalar product of twisted XXX modified Bethe vectors
}

\author{
S. Belliard, N. A. Slavnov ${ }^{\ddagger}$ and B. Vallet ${ }^{\dagger} 1$ \\ † Institut de Physique Théorique, DSM, CEA, URA2306 CNRS Saclay, F-91191, Gif-sur-Yvette, \\ France \\ $¥$ Steklov Mathematical Institute of Russian Academy of Sciences, Moscow, Russia
}

\begin{abstract}
We consider closed XXX spin chains with broken total spin $U(1)$ symmetry within the framework of the modified algebraic Bethe ansatz. We study multiple actions of the modified monodromy matrix entries on the modified Bethe vectors. The obtained formulas of the multiple actions allow us to calculate the scalar products of the modified Bethe vectors. We find an analog of Izergin-Korepin formula for the scalar products. This formula involves modified Izergin determinants and can be expressed as sums over partitions of the Bethe parameters.
\end{abstract}

Keywords: integrable spin chain, algebraic Bethe ansatz, Izergin determinant, scalar product

2010 Mathematics Subject Classification: 82R53; 81R12

\section{Introduction}

The recent development of the algebraic Bethe ansatz (ABA) for the models without $U(1)$ symmetry2, the so called modified algebraic Bethe ansatz (MABA), gives access to the spectrum and associated eigenstates of the models (see [1, 2] for the case of the twisted XXX spin chain and references therein for other models). A further natural task is to calculate the correlation functions within the framework of this method. Development in this direction would allow to adapt the technique of the usual ABA for the study of correlation functions for models with $U(1)$ symmetry [3] to models without $U(1)$ symmetry. In turn, this would allow to obtain exact solutions in a wide range of fields, such as statistical physics, condensed matter physics, high energy physics, mathematical physics, and so on.

In the study of correlation functions within the framework of the ABA, the scalar products of Bethe vectors play an important role [3 9]. Whereas the scalar products are known, one can compute the form factors of local operators [10 20]. In turn, knowing the form factors, it is possible to calculate the correlation functions by means of their form factor expansion [21 29].

\footnotetext{
${ }^{1}$ samuel.belliard@gmail.com, nslavnov@mi.ras.ru, benoit.vallet@u-psud.fr

2 Namely models for which the total spin operator or Cartan operator do not commute with the transfer matrix and the Hamiltonian.
} 
The calculation of the scalar products is based on the formulas for the multiple action 3 of the monodromy matrix entries on the Bethe vectors [30 33]. The specificity of the MABA is that the action of the elements of the monodromy matrix on the highest weight vector is nonstandard. Usually, this vector is an eigenvector of the diagonal elements and it is annihilated by the lowertriangular part of the monodromy matrix. However, the monodromy matrix of MABA is obtained from the usual one by means of a non-diagonal twist transformation. This transformation does not affect the commutation relations between the matrix elements, but changes their actions on the highest weight vector 4 . In particular, the latter is no longer an eigenvector of the diagonal entries of the monodromy matrix. As a result, the multiple actions formulas change significantly.

In this paper we consider $\mathfrak{g l}_{2}$-invariant integrable models. An example of such models is the XXX spin- $\frac{1}{2}$ chain with the Hamiltonian

$$
H=\sum_{k=1}^{N}\left(\sigma_{k}^{x} \otimes \sigma_{k+1}^{x}+\sigma_{k}^{y} \otimes \sigma_{k+1}^{y}+\sigma_{k}^{z} \otimes \sigma_{k+1}^{z}\right),
$$

subject to the following non-diagonal boundary conditions:

$$
\begin{aligned}
\gamma \sigma_{N+1}^{x} & =\frac{\tilde{\kappa}^{2}+\kappa^{2}-\kappa_{+}^{2}-\kappa_{-}^{2}}{2} \sigma_{1}^{x}+i \frac{\kappa^{2}-\tilde{\kappa}^{2}-\kappa_{+}^{2}+\kappa_{-}^{2}}{2} \sigma_{1}^{y}+\left(\kappa \kappa_{-}-\tilde{\kappa} \kappa_{+}\right) \sigma_{1}^{z}, \\
\gamma \sigma_{N+1}^{y} & =i \frac{\tilde{\kappa}^{2}-\kappa^{2}-\kappa_{+}^{2}+\kappa_{-}^{2}}{2} \sigma_{1}^{x}+\frac{\tilde{\kappa}^{2}+\kappa^{2}+\kappa_{+}^{2}+\kappa_{-}^{2}}{2} \sigma_{1}^{y}-i\left(\tilde{\kappa} \kappa_{+}+\kappa \kappa_{-}\right) \sigma_{1}^{z} \\
\gamma \sigma_{N+1}^{z} & =\left(\kappa \kappa_{+}-\tilde{\kappa} \kappa_{-}\right) \sigma_{1}^{x}+i\left(\tilde{\kappa} \kappa_{-}+\kappa \kappa_{+}\right) \sigma_{1}^{y}+\left(\tilde{\kappa} \kappa+\kappa_{+} \kappa_{-}\right) \sigma_{1}^{z} .
\end{aligned}
$$

The twist parameters $\left\{\kappa, \tilde{\kappa}, \kappa_{+}, \kappa_{-}\right\}$are generic complex numbers and $\gamma=\tilde{\kappa} \kappa-\kappa_{+} \kappa_{-}$. The Pauli matrices $5 \sigma_{k}^{\alpha}$, with $\alpha=x, y, z$, act non-trivially on the $k$-th component of the quantum space $\mathcal{H}=\otimes_{j=1}^{N} V_{j}$ with $V_{j}=\mathbb{C}^{2}$. The twist parameters $\left\{\kappa, \tilde{\kappa}, \kappa_{+}, \kappa_{-}\right\}$are the entries of the most general $2 \times 2$ matrix $K$ that will be introduced in the framework of the ABA (see section 5 and also in [1, 2]).

Our consideration is not restricted to the Hamiltonian (1.1) only. Actually, we consider a more general case with arbitrary highest weight representation and arbitrary non-diagonal twist transformation of the monodromy matrix. We find the multiple actions of the modified operators on the modified Bethe vectors. This corresponds to the repeated action of the same operator which depends, in general, on different parameters. This allows us to find a closed expression for the scalar product of two modified Bethe vectors. Multiple action formulas of the usual ABA are expressed in terms of a partition function of the six-vertex model with domain wall boundary condition [4]. This latter has an explicit representation in terms of the Izergin determinant 37]. Within the framework of the MABA one deals with certain deformation of the Izergin determinant that we call a modified Izergin determinant. It depends on the parameters of the modified Bethe vectors, but also on the twist parameters. Remarkably, the multiple action formulas and the scalar products of Bethe vectors, being written in terms of the modified Izergin determinant, have almost the same form as their analogs in the usual ABA.

\footnotetext{
${ }^{3}$ By the multiple action of an operator, we refer to the application of a product of operators of its kind.

${ }^{4}$ A similar transformation occurs on the framework of the so-called $B^{\text {good }}$ operator, see 34 36].

${ }^{5} \sigma^{z}=\left(\begin{array}{cc}1 & 0 \\ 0 & -1\end{array}\right), \quad \sigma^{+}=\left(\begin{array}{ll}0 & 1 \\ 0 & 0\end{array}\right), \quad \sigma^{-}=\left(\begin{array}{ll}0 & 0 \\ 1 & 0\end{array}\right), \quad \sigma^{x}=\sigma^{+}+\sigma^{-}, \quad \sigma^{y}=i\left(\sigma^{-}-\sigma^{+}\right)$.
} 
This paper is organized as follows. In section 2 we recall the main tools of the ABA. In section 3 we introduce our notation and the modified Izergin determinant. We recall multiple actions and a scalar product formula within the standard framework of the ABA in section 4. In section [5 we introduce the modified operators and consider their multiple actions on the modified Bethe vectors. Section 6 is devoted to the calculation of the scalar product of modified Bethe vectors. Auxiliary formulas are gathered in appendices. In appendix $\mathrm{A}$ we list some properties of the modified Izergin determinant. In appendix $\mathrm{B}$ we give simple and multiple commutation relations of the monodromy matrix entries within the standard framework of the ABA. Appendix C contains a description of a special automorphism of the Yangian of $\mathfrak{g l}_{2}$.

\section{Basic notions}

We consider integrable models described by a rational $R$-matrix belonging to $\operatorname{End}\left(\mathbb{C}^{2} \otimes \mathbb{C}^{2}\right)$ :

$$
R(u)=\frac{u}{c} I+P
$$

Here $c$ is a constant, $I=\sum_{i, j=1}^{2} E_{i i} \otimes E_{j j}$ is the identity operator on $\mathbb{C}^{2} \otimes \mathbb{C}^{2}, P=\sum_{i, j=1}^{2} E_{i j} \otimes E_{j i}$ is the permutation operator on $\mathbb{C}^{2} \otimes \mathbb{C}^{2}$, and are elementary unites: $\left(E_{i j}\right)_{k l}=\delta_{i k} \delta_{j l}$. The $R$-matrix (2.1) solves the Yang-Baxter equation

$$
R_{12}(u-v) R_{13}(u-w) R_{23}(v-w)=R_{23}(v-w) R_{13}(u-w) R_{12}(u-v) .
$$

This equation holds in the tensor product $V_{1} \otimes V_{2} \otimes V_{3}$, where each $V_{k} \sim \mathbb{C}^{2}$. The $R$-matrix $R_{i j}$ acts nontrivially in the spaces $V_{i}$ and $V_{j}$, while it acts as the identity operator in the remaining space. The $R$-matrix (2.1) is $\mathfrak{g l}_{2}$-invariant (and therefore, $G L(2)$-invariant), and thus,

$$
\left[R_{a b}(u), K_{a}+K_{b}\right]=\left[R_{a b}(u), K_{a} K_{b}\right]=0,
$$

for any matrix $K \in \operatorname{End}\left(\mathbb{C}^{2}\right)$.

Another important object of the ABA is a monodromy matrix $T(u)$ :

$$
T(u)=\left(\begin{array}{ll}
t_{11}(u) & t_{12}(u) \\
t_{21}(u) & t_{22}(u)
\end{array}\right)
$$

The operator-valued entries of the monodromy matrix $t_{i j}(u)$ depend on the complex $u$ (the spectral parameter) and act in a Hilbert space $\mathcal{H}$ of the associated quantum model. This matrix satisfies an RTT relation

$$
R_{a b}(u-v) T_{a}(u) T_{b}(v)=T_{b}(v) T_{a}(u) R_{a b}(u-v),
$$

which encodes the commutation relations of the entries $t_{i j}(u)$ (see Appendix $\mathbb{B}$ ). These commutation relations generate a quantum group algebra, so called Yangian of $\mathfrak{g l}_{2}$.

We assume that the Hilbert space $\mathcal{H}$ contains the highest weight vector $|0\rangle$ possessing the following properties:

$$
t_{i i}(u)|0\rangle=\lambda_{i}(u)|0\rangle, \quad t_{21}(u)|0\rangle=0 .
$$


Here $\lambda_{i}(u)$ are some complex valued functions. These functions fix a highest weight representation $\mathcal{V}\left(\lambda_{1}(u), \lambda_{2}(u)\right)$ of the RTT-algebra (2.5). The action of the operator $t_{12}$ on $|0\rangle$ is free. A state obtained by the successive action of $t_{12}$ on the highest weight vector is called a Bethe vector:

$$
t_{12}(\bar{v})|0\rangle=\prod_{i=1}^{m} t_{12}\left(v_{i}\right)|0\rangle
$$

where $m=0,1, \ldots$, and $\bar{v}=\left\{v_{1}, \ldots, v_{m}\right\}$.

To study scalar products of Bethe vectors we also use the dual highest weight vector $\langle 0|$ defined by

$$
\langle 0| t_{i i}(u)=\lambda_{i}(u)\langle 0|, \quad\langle 0| t_{12}(u)=0, \quad\langle 0 \mid 0\rangle=1 .
$$

Here the functions $\lambda_{i}(u)$ are the same as in (2.6).

\section{Notation and modified Izergin determinant}

Let us define the rational functions

$$
g(u, v)=\frac{c}{u-v}, \quad f(u, v)=1+g(u, v)=\frac{u-v+c}{u-v}, \quad h(u, v)=\frac{f(u, v)}{g(u, v)}=\frac{u-v+c}{c},
$$

where $c$ is the constant entering the $R$-matrix (2.1). Actually, all these functions depend on the difference of their arguments. However we do not stress this dependence. This will in particular allow us to use a special shorthand notation (see (3.4)). It is easy to see that the functions introduced above possess the following properties:

$$
\left.\chi(u, v)\right|_{c \rightarrow-c}=\chi(v, u), \quad \chi(-u,-v)=\chi(v, u), \quad \chi(u-c, v)=\chi(u, v+c),
$$

where $\chi$ is any of the three functions. One can also convinces himself that

$$
g(u, v-c)=\frac{1}{h(u, v)}, \quad h(u, v+c)=\frac{1}{g(u, v)}, \quad f(u, v+c)=\frac{1}{f(v, u)} .
$$

Below we consider sets of complex parameters and denote them by a bar. For example, $\bar{u}=\left\{u_{1}, \ldots, u_{n}\right\}$. The notation $\bar{u} \pm c$ means that $\pm c$ is added to all the arguments of the set $\bar{u}$. We agree upon that the notation $\bar{u}_{k}$ refers to the set that is complementary in $\bar{u}$ to the element $u_{k}$, that is, $\bar{u}_{k}=\bar{u} \backslash u_{k}$.

To make the formulas more compact, we use a shorthand notation for the products of the rational functions (3.1), the operators $t_{k l}(u)$ (2.4), and their vacuum eigenvalues $\lambda_{i}(u)$ (2.6). Namely, if the function (operator) depends on a set of variables (similarly to (2.7)), then one should take the product with respect to the corresponding set. For example,

$$
t_{k l}(\bar{u})=\prod_{j=1}^{n} t_{k l}\left(u_{j}\right), \quad \lambda_{i}(\bar{u})=\prod_{j=1}^{n} \lambda_{i}\left(u_{j}\right), \quad f(z, \bar{u})=\prod_{j=1}^{n} f\left(z, u_{j}\right), \quad f\left(\bar{u}_{k}, u_{k}\right)=\prod_{\substack{j=1 \\ j \neq k}}^{n} f\left(u_{j}, u_{k}\right),
$$


and so on. Note that due to commutativity of the $t_{k l}$-operators the first product in (3.4) is well defined. Notation $f(\bar{u}, \bar{v})$ means the double product over the sets $\bar{u}$ and $\bar{v}$. By definition any product over the empty set is equal to 1 . A double product is equal to 1 if at least one of the sets is empty.

Later we will extend this convention to the products of matrix elements of the twisted monodromy matrix.

\subsection{Modified Izergin determinant}

In many formulas of the $\mathrm{ABA}$ the Izergin determinant appears [4, 37]. Within the framework of the MABA we have to deal with a deformation of this object that we call a modified Izergin determinant.

Definition 3.1. Let $\bar{u}=\left\{u_{1}, \ldots, u_{n}\right\}, \bar{v}=\left\{v_{1}, \ldots, v_{m}\right\}$ and $z$ be a complex number. Then the modified Izergin determinant $K_{n, m}^{(z)}(\bar{u} \mid \bar{v})$ is defined by

$$
K_{n, m}^{(z)}(\bar{u} \mid \bar{v})=\operatorname{det}_{m}\left(-z \delta_{j k}+\frac{f\left(\bar{u}, v_{j}\right) f\left(v_{j}, \bar{v}_{j}\right)}{h\left(v_{j}, v_{k}\right)}\right) .
$$

Alternatively the modified Izergin determinant can be presented as

$$
K_{n, m}^{(z)}(\bar{u} \mid \bar{v})=(1-z)^{m-n} \operatorname{det}_{n}\left(\delta_{j k} f\left(u_{j}, \bar{v}\right)-z \frac{f\left(u_{j}, \bar{u}_{j}\right)}{h\left(u_{j}, u_{k}\right)}\right) .
$$

The proof of the equivalence of representations (3.5) and (3.6) can be found in proposition 4.1 of [38]. It is based on the recursive property (A.19). The modified Izergin determinant is related to the partial domain wall partition functions [39]. Other correspondences will be discussed elsewhere.

It is also convenient to introduce a conjugated modified Izergin determinant as

$$
\bar{K}_{n, m}^{(z)}(\bar{u} \mid \bar{v})=\left.K_{n, m}^{(z)}(\bar{u} \mid \bar{v})\right|_{c \rightarrow-c}=\operatorname{det}_{m}\left(-z \delta_{j k}+\frac{f\left(v_{j}, \bar{u}\right) f\left(\bar{v}_{j}, v_{j}\right)}{h\left(v_{k}, v_{j}\right)}\right),
$$

or equivalently

$$
\bar{K}_{n, m}^{(z)}(\bar{u} \mid \bar{v})=(1-z)^{m-n} \operatorname{det}_{n}\left(\delta_{j k} f\left(\bar{v}, u_{j}\right)-z \frac{f\left(\bar{u}_{j}, u_{j}\right)}{h\left(u_{k}, u_{j}\right)}\right) .
$$

In the particular case $z=1$ and $\# \bar{u}=\# \bar{v}=n$ the modified Izergin determinant turns into the ordinary Izergin determinant, that we traditionally denote by $K_{n}(\bar{u} \mid \bar{v})$ :

$$
K_{n, n}^{(1)}(\bar{u} \mid \bar{v})=K_{n}(\bar{u} \mid \bar{v}) .
$$

This property can be seen from the recursion (A.19) and the initial condition (A.4). It also follows from (3.6) that

$$
K_{n, m}^{(1)}(\bar{u} \mid \bar{v})=0, \quad \text { for } \quad n<m .
$$

Other properties of the modified Izergin determinant are collected in Appendix A, 


\section{Multiple actions}

Actions of the operators $t_{i j}(u)$ on the Bethe vectors (2.7) were computed in [40] (see also [3]). To study the problem of the scalar products one should calculate multiple actions of the form

$$
t_{i j}(\bar{u}) t_{12}(\bar{v})|0\rangle .
$$

Here, according to the convention on the shorthand notation (3.4) $t_{i j}(\bar{u})$ is the product of the operators $t_{i j}$ over the set $\bar{u}=\left\{u_{1}, \ldots, u_{n}\right\}$. Such multiple actions of the monodromy matrix entries were found in [31] for the models with $\mathfrak{g l}_{3}$-invariant $R$-matrix. In our particular case, these formulas give the multiple actions for models with $\mathfrak{g l}_{2}$-invariant $R$-matrix.

Multiple action formulas are given in terms of sums over partitions of the set $\bar{w}=\{\bar{u}, \bar{v}\}$ into subsets. Here and below we mostly denote the subsets by Roman subscripts (except for some special cases). Notation $\bar{w} \Rightarrow\left\{\bar{w}_{\mathrm{I}}, \bar{w}_{\mathrm{II}}\right\}$ (and similar ones) means that the set $\bar{w}$ is divided into subsets $\bar{w}_{\text {I }}$ and $\bar{w}_{\text {II }}$ such that $\bar{w}_{\text {I }} \cup \bar{w}_{\text {II }}=\bar{w}$ and $\bar{w}_{\text {I }} \cap \bar{w}_{\text {II }}=\emptyset$.

Proposition 4.1. 31] Let $\# \bar{u}=n, \# \bar{v}=m, \bar{w}=\{\bar{u}, \bar{v}\}$, and $K_{n}$ be the Izergin determinant (3.9). Then

$$
t_{12}(\bar{u}) t_{12}(\bar{v})|0\rangle=t_{12}(\bar{w})|0\rangle .
$$

The actions of the diagonal elements $t_{i i}$ are given by

$$
\begin{gathered}
t_{11}(\bar{u}) t_{12}(\bar{v})|0\rangle=(-1)^{n} \sum_{\substack{\bar{w} \Rightarrow\left\{\bar{w}_{\mathrm{I}}, \bar{w}_{\mathrm{II}}\right\} \\
\# \bar{w}_{\mathrm{I}}=n}} \lambda_{1}\left(\bar{w}_{\mathrm{I}}\right) \bar{K}_{n}\left(\bar{u} \mid \bar{w}_{\mathrm{I}}-c\right) f\left(\bar{w}_{\mathrm{II}}, \bar{w}_{\mathrm{I}}\right) t_{12}\left(\bar{w}_{\mathrm{II}}\right)|0\rangle, \\
t_{22}(\bar{u}) t_{12}(\bar{v})|0\rangle=(-1)^{n} \sum_{\substack{\bar{w} \Rightarrow\left\{\bar{w}_{\mathrm{I}}, \bar{w}_{\mathrm{I}}\right\} \\
\# \# \overline{\mathrm{w}}_{\mathrm{I}}=n}} \lambda_{2}\left(\bar{w}_{\mathrm{I}}\right) K_{n}\left(\bar{u} \mid \bar{w}_{\mathrm{I}}+c\right) f\left(\bar{w}_{\mathrm{I}}, \bar{w}_{\mathrm{II}}\right) t_{12}\left(\bar{w}_{\mathrm{II}}\right)|0\rangle,
\end{gathered}
$$

where the sums are taken over partitions ? $\{\bar{u}, \bar{v}\}=\bar{w} \Rightarrow\left\{\bar{w}_{\mathrm{I}}, \bar{w}_{\mathrm{II}}\right\}$ such that $\# \bar{w}_{\mathrm{I}}=n$. The action of the elements $t_{21}$ reads

$$
\begin{array}{r}
t_{21}(\bar{u}) t_{12}(\bar{v})|0\rangle=\sum_{\substack{\bar{w} \Rightarrow\left\{\bar{w}_{\mathrm{I}} \bar{w}_{\text {II }}\right\} \\
\# \bar{w}_{\mathrm{I}}=\# \bar{w}_{\mathrm{II}}=n}} \lambda_{2}\left(\bar{w}_{\mathrm{I}}\right) \lambda_{1}\left(\bar{w}_{\mathrm{II}}\right) K_{n}\left(\bar{u} \mid \bar{w}_{\mathrm{I}}+c\right) \bar{K}_{n}\left(\bar{u} \mid \bar{w}_{\mathrm{II}}-c\right) \\
\times f\left(\bar{w}_{\mathrm{I}}, \bar{w}_{\mathrm{II}}\right) f\left(\bar{w}_{\mathrm{I}}, \bar{w}_{\mathrm{III}}\right) f\left(\bar{w}_{\mathrm{III}}, \bar{w}_{\mathrm{II}}\right) t_{12}\left(\bar{w}_{\mathrm{III}}\right)|0\rangle,
\end{array}
$$

where the sum is taken over partitions $\bar{w} \Rightarrow\left\{\bar{w}_{\mathrm{I}}, \bar{w}_{\mathrm{II}}, \bar{w}_{\mathrm{III}}\right\}$ such that $\# \bar{w}_{\mathrm{I}}=\# \bar{w}_{\mathrm{II}}=n$.

Note that the action formulas (4.3) are the direct consequence of the commutation relations (B.6).

Equation (4.5) gives immediate access to the scalar product of Bethe vectors defined by

$$
S_{t}^{n}(\bar{u}, \bar{v})=\left\langle 0\left|t_{21}(\bar{u}) t_{12}(\bar{v})\right| 0\right\rangle,
$$

where $\# \bar{u}=\# \bar{v}=n$. 
Theorem 4.1. Let $\# \bar{u}=\# \bar{v}=n$. Then the scalar product of two Bethe vectors is given by

$$
S_{t}^{n}(\bar{u}, \bar{v})=\sum_{\substack{\bar{w} \Rightarrow\left\{\bar{w}_{\mathrm{I}}, \bar{w}_{\text {II }}\right\} \\ \# \bar{w}_{\mathrm{I}}=\# \bar{w}_{\mathrm{II}}=n}} \lambda_{2}\left(\bar{w}_{\mathrm{I}}\right) \lambda_{1}\left(\bar{w}_{\mathrm{II}}\right) K_{n}\left(\bar{u} \mid \bar{w}_{\mathrm{I}}+c\right) \bar{K}_{n}\left(\bar{u} \mid \bar{w}_{\mathrm{II}}-c\right) f\left(\bar{w}_{\mathrm{I}}, \bar{w}_{\mathrm{II}}\right) .
$$

where the sum is taken over partitions $\bar{w} \Rightarrow\left\{\bar{w}_{\mathrm{I}}, \bar{w}_{\mathrm{II}}\right\}$ such that $\# \bar{w}_{\mathrm{I}}=\# \bar{w}_{\mathrm{II}}=n$.

The sum (4.7) can also be written in the form of the sum over independent partitions of the sets $\bar{u}$ and $\bar{v}$. Then it corresponds to the Izergin-Korepin formula [3].

Corollary 4.1. Let $\# \bar{u}=\# \bar{v}=n$. Then the scalar product of two Bethe vectors is given by

$$
S_{t}^{n}(\bar{u}, \bar{v})=\sum_{\substack{\bar{u} \Rightarrow\left\{\bar{u}_{\mathrm{I}}, \bar{u}_{\mathrm{II}}\right\} \\ \bar{v} \Rightarrow\left\{\bar{v}_{\mathrm{I}}, \bar{v}_{\mathrm{II}}\right\} \\ \# \overline{\mathrm{I}}_{\mathrm{I}}=\# \bar{v}_{\mathrm{I}}}} \lambda_{2}\left(\bar{u}_{\mathrm{I}}\right) \lambda_{2}\left(\bar{v}_{\mathrm{II}}\right) \lambda_{1}\left(\bar{u}_{\mathrm{II}}\right) \lambda_{1}\left(\bar{v}_{\mathrm{I}}\right) K_{n_{2}}\left(\bar{v}_{\mathrm{II}} \mid \bar{u}_{\mathrm{II}}\right) \bar{K}_{n_{1}}\left(\bar{v}_{\mathrm{I}} \mid \bar{u}_{\mathrm{I}}\right) f\left(\bar{u}_{\mathrm{I}}, \bar{u}_{\mathrm{II}}\right) f\left(\bar{v}_{\mathrm{II}}, \bar{v}_{\mathrm{I}}\right),
$$

where the sum is taken over partitions $\bar{u} \Rightarrow\left\{\bar{u}_{\mathrm{I}}, \bar{u}_{\mathrm{II}}\right\}$ and $\bar{v} \Rightarrow\left\{\bar{v}_{\mathrm{I}}, \bar{v}_{\mathrm{II}}\right\}$ such that $\# \bar{u}_{\mathrm{I}}=\# \bar{v}_{\mathrm{I}}=n_{1}$, $\# \bar{u}_{\mathrm{II}}=\# \bar{v}_{\mathrm{II}}=n_{2}$, where $n_{1}=0,1, \ldots, n$ and $n=n_{1}+n_{2}$.

Proof. We set in (4.7) $\bar{w}_{\mathrm{I}} \Rightarrow\left\{\bar{u}_{\mathrm{I}}, \bar{v}_{\mathrm{II}}\right\}$ and $\bar{w}_{\mathrm{II}} \Rightarrow\left\{\bar{u}_{\mathrm{II}}, \bar{v}_{\mathrm{I}}\right\}$. Let $\# \bar{u}_{\mathrm{I}}=\# \bar{v}_{\mathrm{I}}=n_{1}, \# \bar{u}_{\mathrm{II}}=\# \bar{v}_{\mathrm{II}}=n_{2}$, where $n_{1}=0,1, \ldots, n$ and $n=n_{1}+n_{2}$. Using (A.5) and (A.6) we obtain

$$
\begin{aligned}
& S_{t}^{n}(\bar{u}, \bar{v})=(-1)^{n} \sum_{\substack{\bar{u} \Rightarrow\left\{\bar{u}_{\mathrm{I}}, \bar{u}_{\mathrm{II}}\right\} \\
\bar{v} \Rightarrow\left\{\bar{v}_{\mathrm{I}}, \bar{v}_{\mathrm{II}}\right\} \\
\# \bar{u}_{\mathrm{I}}=\# \bar{v}_{\mathrm{I}}}} \lambda_{2}\left(\bar{u}_{\mathrm{I}}\right) \lambda_{2}\left(\bar{v}_{\mathrm{II}}\right) \lambda_{1}\left(\bar{u}_{\mathrm{II}}\right) \lambda_{1}\left(\bar{v}_{\mathrm{I}}\right) K_{n_{2}}\left(\bar{u}_{\mathrm{II}} \mid \bar{v}_{\mathrm{II}}+c\right) \bar{K}_{n_{1}}\left(\bar{u}_{\mathrm{I}} \mid \bar{v}_{\mathrm{I}}-c\right) \\
& \times f\left(\bar{u}_{\mathrm{I}}, \bar{u}_{\mathrm{II}}\right) f\left(\bar{v}_{\mathrm{II}}, \bar{v}_{\mathrm{I}}\right) f\left(\bar{u}_{\mathrm{I}}, \bar{v}_{\mathrm{I}}\right) f\left(\bar{v}_{\mathrm{II}}, \bar{u}_{\mathrm{II}}\right) .
\end{aligned}
$$

Then the use of (A.15) and (A.16) immediately leads us to (4.8).

\section{Multiple actions of modified operators on Bethe vectors}

A monodromy matrix of MABA is constructed as a twist transformation of the original monodromy matrix (2.4). In [1, 2] we discussed the factorisation of the twist matrix $K=B D A$ (where $D$ is a diagonal matrix), which allows us to use the MABA. It includes some freedom by the transformation $A \rightarrow S A$ and $B \rightarrow B S^{-1}$ for any invertible diagonal matrix $S$. Let us consider the following parametrization of the two matrices $A$ and $B$ :

$$
A=\sqrt{\mu}\left(\begin{array}{ll}
1 & \frac{\rho_{2}}{\kappa^{-}} \\
\frac{\rho_{1}}{\kappa^{+}} & 1
\end{array}\right), \quad B=\sqrt{\mu}\left(\begin{array}{ll}
1 & \frac{\rho_{1}}{\kappa^{-}} \\
\frac{\rho_{2}}{\kappa^{+}} & 1
\end{array}\right), \quad \mu=\frac{1}{1-\frac{\rho_{1} \rho_{2}}{\kappa^{+} \kappa^{-}}} .
$$

Here $\rho_{i}$ and $\kappa^{ \pm}$are generic parameters. Due to the property (2.3), the transformation of the monodromy matrix

$$
\bar{T}(u)=A T(u) B=\left(\begin{array}{ll}
\nu_{11}(u) & \nu_{12}(u) \\
\nu_{21}(u) & \nu_{22}(u)
\end{array}\right)
$$


is an automorphism of the Yangian of $\mathfrak{g l}_{2}$, i.e. new operators $\nu_{i j}$ satisfy the same commutation relations as the $t_{i j}(z)$, given in Appendix $\mathbb{B}$. However, the actions of the modified operators $\left\{\nu_{i i}(u), \nu_{21}(u)\right\}$ on the highest weight vector (2.6) change. It is easy to see that now they are given by

$$
\begin{aligned}
& \nu_{11}(u)|0\rangle=\lambda_{1}(u)|0\rangle+\beta_{2} \nu_{12}(u)|0\rangle, \\
& \nu_{22}(u)|0\rangle=\lambda_{2}(u)|0\rangle+\beta_{1} \nu_{12}(u)|0\rangle, \\
& \nu_{21}(u)|0\rangle=\left(\beta_{1} \lambda_{1}(u)+\beta_{2} \lambda_{2}(u)\right)|0\rangle+\beta_{1} \beta_{2} \nu_{12}(u)|0\rangle,
\end{aligned}
$$

where $\beta_{i}=\frac{\rho_{i}}{\kappa^{+}}$.

The modified Bethe vectors are given by

$$
\nu_{12}(\bar{v})|0\rangle=\prod_{i=1}^{m} \nu_{12}\left(v_{i}\right)|0\rangle
$$

with $m=0,1, \ldots$. Here we extended the convention on the shorthand notation (3.4) to the products of the operators $\nu_{i j}$. Since the commutation relations of $\nu_{i j}$ are the same as the ones of $t_{i j}$, we have, in particular, $\left[\nu_{i j}(u), \nu_{i j}(v)\right]=0$. Thus, the products $\nu_{i j}(\bar{v})$ are well defined.

\subsection{Multiple actions of the modified diagonal operators}

It is clear that changing the action on the highest weight vector leads to a modification of the multiple action formulas.

Proposition 5.1. The multiple actions of the products of the diagonal modified operators $\nu_{i i}(\bar{u})$, with $\bar{u}=\left\{u_{1}, \ldots, u_{n}\right\}$, on the modified Bethe vector $\nu_{12}(\bar{v})|0\rangle$, with $\bar{v}=\left\{v_{1}, \ldots, v_{m}\right\}$, are given by

$$
\begin{aligned}
& \nu_{11}(\bar{u}) \nu_{12}(\bar{v})|0\rangle=\beta_{2}^{n} \sum_{\bar{w} \Rightarrow\left\{\bar{w}_{\mathrm{I}}, \bar{w}_{\text {II }}\right\}}\left(-\beta_{2}\right)^{-l} \lambda_{1}\left(\bar{w}_{\mathrm{I}}\right) \bar{K}_{n, l}^{(1)}\left(\bar{u} \mid \bar{w}_{\mathrm{I}}-c\right) f\left(\bar{w}_{\mathrm{II}}, \bar{w}_{\mathrm{I}}\right) \nu_{12}\left(\bar{w}_{\mathrm{II}}\right)|0\rangle, \\
& \nu_{22}(\bar{u}) \nu_{12}(\bar{v})|0\rangle=\beta_{1}^{n} \sum_{\bar{w} \Rightarrow\left\{\bar{w}_{\mathrm{I}}, \bar{w}_{\mathrm{II}}\right\}}\left(-\beta_{1}\right)^{-l} \lambda_{2}\left(\bar{w}_{\mathrm{I}}\right) K_{n, l}^{(1)}\left(\bar{u}_{\mid} \mid \bar{w}_{\mathrm{I}}+c\right) f\left(\bar{w}_{\mathrm{I}}, \bar{w}_{\mathrm{II}}\right) \nu_{12}\left(\bar{w}_{\mathrm{II}}\right)|0\rangle .
\end{aligned}
$$

Here $l=\# \bar{w}_{\mathrm{I}}$. The sum is taken over all partitions $\{\bar{u}, \bar{v}\}=\bar{w} \Rightarrow\left\{\bar{w}_{\mathrm{I}}, \bar{w}_{\mathrm{II}}\right\}$. There is no restrictions on the cardinalities of the subsets. The function $K_{n, l}^{(1)}$ and $\bar{K}_{n, l}^{(1)}$ respectively are the modified Izergin determinants (3.5) and (3.7) at $z=1$.

Remark 5.1. The main difference between modified action formulas and equations (4.3) is the replacement of the ordinary Izergin determinants with the modified Izergin determinants. This leads to the fact that there is no restriction on the cardinalities of the subsets in formulas (5.7), (5.8). However, due to the property $K_{n, l}^{(1)}(\bar{u} \mid \bar{v})=\bar{K}_{n, l}^{(1)}(\bar{u} \mid \bar{v})=0$ for $n<l$, the summation in (5.7), (5.8) is carried out only over those partitions for which $l \leq n$.

Proof. We give a detailed proof of formula (5.7). The proof of formula (5.8) is completely analogous. It also follows form (5.7) due to the symmetry of the Yangian described in appendix C. 
We first consider the case $n=\# \bar{u}=1$. In fact, in this case, equation (5.7) was firstly conjectured in [1] and then proved in [35]. Therefore, we consider this case for the sake of completeness only.

Since the operators $\nu_{i j}$ possess the same commutation relations as $t_{i j}$, we can use (B.6) for $n=1$ :

$$
\nu_{11}(u) \nu_{12}(\bar{v})=-\sum_{\substack{\bar{w} \Rightarrow\left\{\bar{w}_{\mathrm{I}}, \bar{w}_{\text {II }}\right\} \\ \# \bar{w}_{\mathrm{I}}=1}} \bar{K}_{1}\left(u \mid \bar{w}_{\mathrm{I}}-c\right) f\left(\bar{w}_{\text {II }}, \bar{w}_{\mathrm{I}}\right) \nu_{12}\left(\bar{w}_{\text {II }}\right) \nu_{11}\left(\bar{w}_{\mathrm{I}}\right) .
$$

Here $\bar{w}=\{u, \bar{v}\}$. The sum is taken over partitions $\bar{w} \Rightarrow\left\{\bar{w}_{\mathrm{I}}, \bar{w}_{\mathrm{II}}\right\}$ such that $\# \bar{w}_{\mathrm{I}}=1$. Applying this equation to $|0\rangle$ and using (5.3) we obtain

$$
\nu_{11}(u) \nu_{12}(\bar{v})|0\rangle=-\sum_{\substack{\bar{w} \Rightarrow\left\{\bar{w}_{\mathrm{I}}, \bar{w}_{\mathrm{II}}\right\} \\ \# \bar{w}_{\mathrm{I}}=1}} \bar{K}_{1}\left(u \mid \bar{w}_{\mathrm{I}}-c\right) f\left(\bar{w}_{\mathrm{II}}, \bar{w}_{\mathrm{I}}\right) \nu_{12}\left(\bar{w}_{\mathrm{II}}\right)\left(\lambda_{1}\left(\bar{w}_{\mathrm{I}}\right)+\beta_{2} \nu_{12}\left(\bar{w}_{\mathrm{I}}\right)\right)|0\rangle .
$$

The sum over partitions in the term proportional to $\beta_{2}$ can be computed explicitly. Indeed, we have

Then

$$
-\bar{K}_{1}\left(u \mid \bar{w}_{\mathrm{I}}-c\right)=\frac{c}{u-\bar{w}_{\mathrm{I}}+c}=\frac{1}{h\left(u, \bar{w}_{\mathrm{I}}\right)} .
$$

$$
-\beta_{2} \sum_{\substack{\bar{w} \Rightarrow\left\{\bar{w}_{\mathrm{I}}, \bar{w}_{\mathrm{II}}\right\} \\ \# \bar{w}_{\mathrm{I}}=1}} \bar{K}_{1}\left(u \mid \bar{w}_{\mathrm{I}}-c\right) f\left(\bar{w}_{\mathrm{II}}, \bar{w}_{\mathrm{I}}\right) \nu_{12}\left(\bar{w}_{\mathrm{II}}\right) \nu_{12}\left(\bar{w}_{\mathrm{I}}\right)|0\rangle=\beta_{2} \nu_{12}(\bar{w})|0\rangle G
$$

where

$$
G=\sum_{\substack{\bar{w} \Rightarrow\left\{\bar{w}_{\mathrm{I}}, \bar{w}_{\mathrm{II}}\right\} \\ \# \bar{w}_{\mathrm{I}}=1}} \frac{f\left(\bar{w}_{\mathrm{II}}, \bar{w}_{\mathrm{I}}\right)}{h\left(u, \bar{w}_{\mathrm{I}}\right)}
$$

To calculate the sum over partitions (5.12) it is enough to present it as a contour integral

$$
G=\frac{-1}{2 \pi i c} \oint_{|z|=R \rightarrow \infty} \frac{f(\bar{w}, z)}{h(u, z)} d z .
$$

Taking the residue at infinity we obtain $6=1$. Thus,

$$
\nu_{11}(u) \nu_{12}(\bar{v})|0\rangle=\beta_{2} \nu_{12}(\bar{w})|0\rangle-\sum_{\substack{\bar{w} \Rightarrow\left\{\bar{w}_{\mathrm{I}}, \bar{w}_{\mathrm{II}}\right\} \\ \# \bar{w}_{\mathrm{I}}=1}} \lambda_{1}\left(\bar{w}_{\mathrm{I}}\right) \bar{K}_{1}\left(u \mid \bar{w}_{\mathrm{I}}-c\right) f\left(\bar{w}_{\mathrm{II}}, \bar{w}_{\mathrm{I}}\right) \nu_{12}\left(\bar{w}_{\mathrm{II}}\right)|0\rangle .
$$

It remains to compare the result obtained with equation (5.7) for $n=1$. In this case either $l=0$ or $l=1$. It is easy to see that the first term in (5.14) corresponds to the case $l=0$, while the second term gives the sum over partitions for $l=1$. Thus, the action (5.7) is proved for $n=1$.

To proceed further we use induction over $n$. Assume that (5.7) holds for some $n-1$. Then the action of $\nu_{11}(\bar{u})$ on the modified Bethe vector $\nu_{12}(\bar{v})|0\rangle$ can be computed as the successive action of $\nu_{11}\left(\bar{u}_{n}\right)$ and $\nu_{11}\left(u_{n}\right)$ (recall that $\left.\bar{u}_{n}=\bar{u} \backslash u_{n}\right)$. At the first step we have

$$
\nu_{11}(\bar{u}) \nu_{12}(\bar{v})|0\rangle=\nu_{11}\left(u_{n}\right) \beta_{2}^{n-1} \sum_{\bar{\xi} \Rightarrow\left\{\bar{\xi}_{\mathrm{I}}, \bar{\xi}_{\mathrm{II}}\right\}}\left(-\beta_{2}\right)^{-l_{\mathrm{I}}} \lambda_{1}\left(\bar{\xi}_{\mathrm{I}}\right) \bar{K}_{n-1, l}^{(1)}\left(\bar{u}_{n} \mid \bar{\xi}_{\mathrm{I}}-c\right) f\left(\bar{\xi}_{\mathrm{I}}, \bar{\xi}_{\mathrm{I}}\right) \nu_{12}\left(\bar{\xi}_{\mathrm{II}}\right)|0\rangle .
$$

\footnotetext{
${ }^{6}$ Recall that $u \in \bar{w}$, and thus, there is no pole at $z=u+c$.
} 
Here $\bar{\xi}=\left\{\bar{u}_{n}, \bar{v}\right\}$. The sum is taken over partitions $\bar{\xi} \Rightarrow\left\{\bar{\xi}_{\mathrm{I}}, \bar{\xi}_{\mathrm{I}}\right\}$, and $l_{\mathrm{I}}=\# \bar{\xi}_{\mathrm{I}}$. Acting with $\nu_{11}\left(u_{n}\right)$ on the vector $\nu_{12}\left(\bar{\xi}_{\mathrm{II}}\right)|0\rangle$ we obtain

$$
\begin{aligned}
\nu_{11}(\bar{u}) \nu_{12}(\bar{v})|0\rangle=\beta_{2}^{n} \sum_{\bar{\xi} \Rightarrow\left\{\bar{\xi}_{\mathrm{I}}, \bar{\xi}_{\mathrm{II}}\right\}}\left(-\beta_{2}\right)^{-l_{\mathrm{I}}} \lambda_{1}\left(\bar{\xi}_{\mathrm{I}}\right) \bar{K}_{n-1, l_{\mathrm{I}}}^{(1)}\left(\bar{u}_{n} \mid \bar{\xi}_{\mathrm{I}}-c\right) f\left(\bar{\xi}_{\mathrm{II}}, \bar{\xi}_{\mathrm{I}}\right) \\
\times \sum_{\bar{\eta} \Rightarrow\left\{\bar{\eta}_{\mathrm{I}}, \bar{\eta}_{\mathrm{I}}\right\}}\left(-\beta_{2}\right)^{-k_{\mathrm{I}}} \lambda_{1}\left(\bar{\eta}_{\mathrm{I}}\right) \bar{K}_{1, k_{\mathrm{I}}}^{(1)}\left(u_{n} \mid \bar{\eta}_{\mathrm{I}}-c\right) f\left(\bar{\eta}_{\mathrm{II}}, \bar{\eta}_{\mathrm{I}}\right) \nu_{12}\left(\bar{\eta}_{\mathrm{II}}\right)|0\rangle .
\end{aligned}
$$

Here we have one more sum over partitions of the set $\bar{\eta}=\left\{u_{n}, \bar{\xi}_{\mathrm{II}}\right\} \Rightarrow\left\{\bar{\eta}_{\mathrm{I}}, \bar{\eta}_{\mathrm{I}}\right\}$, and $k_{\mathrm{I}}=\# \bar{\eta}_{\mathrm{I}}$.

Thus, in (5.16), the set $\{\bar{u}, \bar{v}\}$ eventually is divided into three subsets $\bar{\xi}_{\mathrm{I}}, \bar{\eta}_{\mathrm{I}}$, and $\bar{\eta}_{\mathrm{I}}$. The subset $\bar{\xi}_{\text {II }}$ plays an intermediate role and should be understood as $\bar{\xi}_{\mathrm{II}}=\left\{\bar{\eta}_{\mathrm{I}}, \bar{\eta}_{\mathrm{II}}\right\} \backslash\left\{u_{n}\right\}$. The only restriction on these partitions is that $u_{n} \notin \bar{\xi}_{\mathrm{I}}$.

Let $\bar{w}=\{\bar{u}, \bar{v}\}$. Denote $\bar{\xi}_{\mathrm{I}}=\bar{w}_{\mathrm{I}}, \bar{\eta}_{\mathrm{I}}=\bar{w}_{\mathrm{II}}$, and $\bar{\eta}_{\mathrm{II}}=\bar{w}_{\mathrm{III}}$. Then $\bar{\xi}_{\mathrm{II}}=\left\{\bar{w}_{\mathrm{II}}, \bar{w}_{\mathrm{III}}\right\} \backslash\left\{u_{n}\right\}$ and

$$
f\left(\bar{\xi}_{\mathrm{II}}, \bar{\xi}_{\mathrm{I}}\right)=\frac{f\left(\bar{w}_{\mathrm{II}}, \bar{w}_{\mathrm{I}}\right) f\left(\bar{w}_{\mathrm{II}}, \bar{w}_{\mathrm{I}}\right)}{f\left(u_{n}, \bar{w}_{\mathrm{I}}\right)} .
$$

Observe that the right hand side of (5.17) vanishes as soon as $u_{n} \in \bar{w}_{\mathrm{I}}$. Thus, the condition $u_{n} \notin \bar{\xi}_{\mathrm{I}}$ holds automatically. Equation (5.16) then takes the following form:

$$
\begin{aligned}
& \nu_{11}(\bar{u}) \nu_{12}(\bar{v})|0\rangle=\beta_{2}^{n} \sum_{\bar{w} \Rightarrow\left\{\bar{w}_{\mathrm{I}}, \bar{w}_{\mathrm{II}}, \bar{w}_{\mathrm{III}}\right\}}\left(-\beta_{2}\right)^{-r_{\mathrm{I}}-r_{\mathrm{II}}} \lambda_{1}\left(\bar{w}_{\mathrm{I}}\right) \lambda_{1}\left(\bar{w}_{\mathrm{II}}\right) \\
& \times \bar{K}_{n-1, r_{\mathrm{I}}}^{(1)}\left(\bar{u}_{n} \mid \bar{w}_{\mathrm{I}}-c\right) \bar{K}_{1, r_{\mathrm{II}}}^{(1)}\left(u_{n} \mid \bar{w}_{\mathrm{II}}-c\right) \frac{f\left(\bar{w}_{\mathrm{II}}, \bar{w}_{\mathrm{I}}\right) f\left(\bar{w}_{\mathrm{II}}, \bar{w}_{\mathrm{I}}\right) f\left(\bar{w}_{\mathrm{III}}, \bar{w}_{\mathrm{II}}\right)}{f\left(u_{n}, \bar{w}_{\mathrm{I}}\right)} \nu_{12}\left(\bar{w}_{\mathrm{III}}\right)|0\rangle .
\end{aligned}
$$

Here $r_{\mathrm{I}}=\# \bar{w}_{\mathrm{I}}$ and $r_{\mathrm{II}}=\# \bar{w}_{\mathrm{II}}$. Let $\left\{\bar{w}_{\mathrm{I}}, \bar{w}_{\mathrm{II}}\right\}=\bar{w}_{0}$ and $r_{0}=\# \bar{w}_{0}$. Then, we recast (5.18) as follows:

$$
\begin{aligned}
\nu_{11}(\bar{u}) \nu_{12}(\bar{v})|0\rangle=\beta_{2}^{n} \sum_{\bar{w} \Rightarrow\left\{\bar{w}_{0}, \bar{w}_{\mathrm{III}}\right\}}\left(-\beta_{2}\right)^{-r_{0}} \lambda_{1}\left(\bar{w}_{0}\right) f\left(\bar{w}_{\mathrm{II}}, \bar{w}_{0}\right) \nu_{12}\left(\bar{w}_{\mathrm{III}}\right)|0\rangle \\
\times \sum_{\bar{w}_{0} \Rightarrow\left\{\bar{w}_{\mathrm{I}}, \bar{w}_{\mathrm{II}}\right\}} \bar{K}_{n-1, r_{\mathrm{I}}}^{(1)}\left(\bar{u}_{n} \mid \bar{w}_{\mathrm{I}}-c\right) \bar{K}_{1, r_{\mathrm{II}}}^{(1)}\left(u_{n} \mid \bar{w}_{\mathrm{II}}-c\right) \frac{f\left(\bar{w}_{\mathrm{II}}, \bar{w}_{\mathrm{I}}\right)}{f\left(u_{n}, \bar{w}_{\mathrm{I}}\right)} .
\end{aligned}
$$

The sum over partitions is now organized in two steps. First, the set $\bar{w}$ is divided into two subsets $\bar{w}_{0} \Rightarrow\left\{\bar{w}_{\mathrm{I}}, \bar{w}_{\mathrm{II}}\right\}$. Then the subset $\bar{w}_{0}$ is divided once more as $\bar{w}_{0} \Rightarrow\left\{\bar{w}_{\mathrm{I}}, \bar{w}_{\mathrm{II}}\right\}$. It is easy to see that the sum over partitions in the second line of (5.19) reduces to the modified Izergin determinant due to (A.29):

$$
\sum_{\bar{w}_{0} \Rightarrow\left\{\bar{w}_{\mathrm{I}}, \bar{w}_{\Pi}\right\}} \bar{K}_{n-1, r_{\mathrm{I}}}^{(1)}\left(\bar{u}_{n} \mid \bar{w}_{\mathrm{I}}-c\right) \bar{K}_{1, r_{\mathrm{II}}}^{(1)}\left(u_{n} \mid \bar{w}_{\mathrm{II}}-c\right) \frac{f\left(\bar{w}_{\mathrm{II}}, \bar{w}_{\mathrm{I}}\right)}{f\left(u_{n}, \bar{w}_{\mathrm{I}}\right)}=\bar{K}_{n, r_{0}}^{(1)}\left(\bar{u} \mid \bar{w}_{0}-c\right) .
$$

Thus, we arrive at

$$
\nu_{11}(\bar{u}) \nu_{12}(\bar{v})|0\rangle=\beta_{2}^{n} \sum_{\bar{w} \Rightarrow\left\{\bar{w}_{0}, \bar{w}_{\text {III }}\right\}}\left(-\beta_{2}\right)^{-r_{0}} \lambda_{1}\left(\bar{w}_{0}\right) \bar{K}_{n, r_{0}}^{(1)}\left(\bar{u} \mid \bar{w}_{0}-c\right) f\left(\bar{w}_{\text {III }}, \bar{w}_{0}\right) \nu_{12}\left(\bar{w}_{\mathrm{III}}\right)|0\rangle .
$$

This equation coincides with (5.7) for $\# \bar{u}=n$ up to the labels of the subsets. 


\subsection{Multiple action of the modified operator $\nu_{21}$}

Proposition 5.2. The multiple action of the product of modified operators $\nu_{21}(\bar{u})$, with $\bar{u}=$ $\left\{u_{1}, \ldots, u_{n}\right\}$, on the modified Bethe vector $\nu_{12}(\bar{v})|0\rangle$, with $\bar{v}=\left\{v_{1}, \ldots, v_{m}\right\}$, is given by

$$
\begin{aligned}
\nu_{21}(\bar{u}) \nu_{12}(\bar{v})|0\rangle= & \sum_{\bar{w} \Rightarrow\left\{\bar{w}_{\mathrm{I}}, \bar{w}_{\mathrm{II}}, \bar{w}_{\mathrm{II}}\right\}}\left(-\beta_{1}\right)^{n-l_{\mathrm{I}}}\left(-\beta_{2}\right)^{n-l_{\mathrm{II}}} \lambda_{2}\left(\bar{w}_{\mathrm{I}}\right) \lambda_{1}\left(\bar{w}_{\mathrm{II}}\right) \\
& \times K_{n, l_{\mathrm{I}}}^{(1)}\left(\bar{u}_{\mid} \mid \bar{w}_{\mathrm{I}}+c\right) \bar{K}_{n, l_{\mathrm{II}}}^{(1)}\left(\bar{u} \mid \bar{w}_{\mathrm{II}}-c\right) f\left(\bar{w}_{\mathrm{I}}, \bar{w}_{\mathrm{II}}\right) f\left(\bar{w}_{\mathrm{I}}, \bar{w}_{\mathrm{II}}\right) f\left(\bar{w}_{\mathrm{III}}, \bar{w}_{\mathrm{II}}\right) \nu_{12}\left(\bar{w}_{\mathrm{II}}\right)|0\rangle .
\end{aligned}
$$

Here $l_{\mathrm{I}}=\bar{w}_{\mathrm{I}}$ and $l_{\mathrm{II}}=\bar{w}_{\mathrm{II}}$. The sum is taken over all partitions $\{\bar{u}, \bar{v}\}=\bar{w} \Rightarrow\left\{\bar{w}_{\mathrm{I}}, \bar{w}_{\mathrm{II}}, \bar{w}_{\mathrm{II}}\right\}$. The function $K_{n, l_{\mathrm{I}}}^{(1)}$ and $\bar{K}_{n, l_{\mathrm{I}}}^{(1)}$ respectively are the modified Izergin determinants (3.5) and (3.7) at $z=1$.

Proof. To prove (5.22) we first use induction over $m=\# \bar{v}$ and then over $n=\# \bar{u}$.

Let $n=1$ and, hence, $\bar{u}=u$. Note that in spite of the sum in (5.22) is taken over all possible partitions of the set $\bar{w}=\{u, \bar{v}\}$, in fact, it is restricted by the condition $l_{i} \leq n$ ( $i=\mathrm{I}$, I $)$, because otherwise the modified Izergin determinants vanish. Thus, for $n=1$ the cardinalities of the subsets $\bar{w}_{\mathrm{I}}$ and $\bar{w}_{\mathrm{II}}$ are either 0 or 1 . Then, it is easy to see that for $n=1$ and $m=0$, equation (5.22) coincides with the action formula (5.5).

Assume that (5.22) holds for some $m-1$, where $m>0$. Using commutation relation (B.5) we obtain

$$
\nu_{21}(u) \nu_{12}(\bar{v})|0\rangle=\left[\nu_{12}\left(v_{m}\right) \nu_{21}(u)+g\left(u, v_{m}\right)\left(\nu_{11}\left(v_{m}\right) \nu_{22}(u)-\nu_{11}(u) \nu_{22}\left(v_{m}\right)\right)\right] \nu_{12}\left(\bar{v}_{m}\right)|0\rangle .
$$

Let us first consider the contribution of the term $\nu_{12}\left(v_{m}\right) \nu_{21}(u)$. Due to the induction assumption we have

$$
\begin{aligned}
\nu_{12}\left(v_{m}\right) \nu_{21}(u) \nu_{12}\left(\bar{v}_{m}\right)|0\rangle= & \sum_{\bar{\xi} \Rightarrow\left\{\bar{\xi}_{\mathrm{I}}, \bar{\xi}_{\mathrm{I}}, \bar{\xi}_{\mathrm{III}}\right\}}\left(-\beta_{1}\right)^{1-l_{\mathrm{I}}}\left(-\beta_{2}\right)^{1-l_{\mathrm{II}}} f\left(\bar{\xi}_{\mathrm{I}}, \bar{\xi}_{\mathrm{II}}\right) f\left(\bar{\xi}_{\mathrm{I}}, \bar{\xi}_{\mathrm{III}}\right) f\left(\bar{\xi}_{\mathrm{II}}, \bar{\xi}_{\mathrm{II}}\right) \\
& \times \lambda_{2}\left(\bar{\xi}_{\mathrm{I}}\right) \lambda_{1}\left(\bar{\xi}_{\mathrm{II}}\right) K_{1, l_{\mathrm{I}}}^{(1)}\left(u \mid \bar{\xi}_{\mathrm{I}}+c\right) \bar{K}_{1, l_{\mathrm{II}}}^{(1)}\left(u \mid \bar{\xi}_{\mathrm{II}}-c\right) \nu_{12}\left(\left\{v_{m}, \bar{\xi}_{\mathrm{II}}\right\}\right)|0\rangle,
\end{aligned}
$$

where $\bar{\xi}=\left\{u, \bar{v}_{m}\right\}$. Let $\bar{w}=\{u, \bar{v}\}$. Then equation (5.24) is equivalent to

$$
\begin{aligned}
& \nu_{12}\left(v_{m}\right) \nu_{21}(u) \nu_{12}\left(\bar{v}_{m}\right)|0\rangle=\sum_{\bar{w} \Rightarrow\left\{\bar{w}_{\mathrm{I}}, \bar{w}_{\mathrm{II}}, \bar{w}_{\mathrm{II}}\right\}}\left(-\beta_{1}\right)^{1-l_{\mathrm{I}}}\left(-\beta_{2}\right)^{1-l_{\mathrm{II}}} \frac{f\left(\bar{w}_{\mathrm{I}}, \bar{w}_{\mathrm{II}}\right) f\left(\bar{w}_{\mathrm{I}}, \bar{w}_{\mathrm{III}}\right) f\left(\bar{w}_{\mathrm{II}}, \bar{w}_{\mathrm{II}}\right)}{f\left(\bar{w}_{\mathrm{I}}, v_{m}\right) f\left(v_{m}, \bar{w}_{\mathrm{II}}\right)} \\
& \times \lambda_{2}\left(\bar{w}_{\mathrm{I}}\right) \lambda_{1}\left(\bar{w}_{\mathrm{II}}\right) K_{1, l_{\mathrm{I}}}^{(1)}\left(u \mid \bar{w}_{\mathrm{I}}+c\right) \bar{K}_{1, l_{\mathrm{II}}}^{(1)}\left(u \mid \bar{w}_{\text {II }}-c\right) \nu_{12}\left(\bar{w}_{\mathrm{III}}\right)|0\rangle .
\end{aligned}
$$

Indeed, due to the factor $\left(f\left(\bar{w}_{\mathrm{I}}, v_{m}\right) f\left(v_{m}, \bar{w}_{\mathrm{II}}\right)\right)^{-1}$ we have $v_{m} \notin \bar{w}_{\mathrm{I}}$ and $v_{m} \notin \bar{w}_{\mathrm{II}}$, because otherwise the corresponding contribution vanishes. Thus, $v_{m} \in \bar{w}_{\mathrm{III}}$. Setting $\bar{w}_{\mathrm{I}}=\bar{\xi}_{\mathrm{I}}, \bar{w}_{\mathrm{II}}=\bar{\xi}_{\mathrm{II}}$, and $\bar{w}_{\mathrm{III}}=$ $\left\{v_{m}, \bar{\xi}_{\mathrm{III}}\right\}$ in (5.25) we immediately arrive at (5.24).

The action of the terms $\nu_{11}\left(v_{m}\right) \nu_{22}(u)$ and $\nu_{11}(u) \nu_{22}\left(v_{m}\right)$ in (5.23) can be computed using Proposition [5.1. We omit simple but rather exhausting intermediate calculations and give the final 
result:

$$
\begin{gathered}
g\left(v_{m}, u\right)\left(\nu_{11}(u) \nu_{22}\left(v_{m}\right)-\nu_{11}\left(v_{m}\right) \nu_{22}(u)\right) \nu\left(\bar{v}_{m}\right)|0\rangle=\sum_{\bar{w} \Rightarrow\left\{\bar{w}_{\mathrm{I}}, \bar{w}_{\mathrm{II}}, \bar{w}_{\mathrm{III}}\right\}}\left(-\beta_{1}\right)^{1-l_{\mathrm{I}}}\left(-\beta_{2}\right)^{1-l_{\mathrm{II}}} \\
\times f\left(\bar{w}_{\mathrm{I}}, \bar{w}_{\mathrm{II}}\right) f\left(\bar{w}_{\mathrm{I}}, \bar{w}_{\mathrm{III}}\right) f\left(\bar{w}_{\mathrm{II}}, \bar{w}_{\mathrm{II}}\right) K_{1, l_{\mathrm{I}}}^{(1)}\left(u \mid \bar{w}_{\mathrm{I}}+c\right) \bar{K}_{1, l_{\mathrm{II}}}^{(1)}\left(u \mid \bar{w}_{\mathrm{II}}-c\right) \\
\times\left(1-\frac{1}{f\left(\bar{w}_{\mathrm{I}}, v_{m}\right) f\left(v_{m}, \bar{w}_{\mathrm{II}}\right)}\right) \lambda_{2}\left(\bar{w}_{\mathrm{I}}\right) \lambda_{1}\left(\bar{w}_{\mathrm{II}}\right) \nu_{12}\left(\bar{w}_{\mathrm{III}}\right)|0\rangle .
\end{gathered}
$$

Combining equations (5.25) and (5.26) we obtain (5.22) for $\# \bar{v}=m$. Thus, the first step of induction is completed.

Let now assume that (5.22) holds for some $n-1$. We prove that then it holds for $\# \bar{u}=n$. The proof is very similar to the one of proposition 5.1, however, it is more bulky.

We act successively as $\nu_{21}(\bar{u})=\nu_{21}\left(u_{n}\right) \nu_{21}\left(\bar{u}_{n}\right)$. Then

$$
\begin{aligned}
\nu_{21}(\bar{u}) \nu_{12}(\bar{v})|0\rangle & =\nu_{21}\left(u_{n}\right) \sum_{\bar{\xi} \Rightarrow\left\{\bar{\xi}_{\mathrm{I}}, \bar{\xi}_{\mathrm{II}}, \bar{\xi}_{\mathrm{III}}\right\}}\left(-\beta_{1}\right)^{n-1-l_{\mathrm{I}}}\left(-\beta_{2}\right)^{n-1-l_{\mathrm{II}}} \lambda_{2}\left(\bar{\xi}_{\mathrm{I}}\right) \lambda_{1}\left(\bar{\xi}_{\mathrm{II}}\right) \\
& \times f\left(\bar{\xi}_{\mathrm{I}}, \bar{\xi}_{\mathrm{II}}\right) f\left(\bar{\xi}_{\mathrm{I}}, \bar{\xi}_{\mathrm{II}}\right) f\left(\bar{\xi}_{\mathrm{II}}, \bar{\xi}_{\mathrm{II}}\right) K_{n-1, l_{\mathrm{I}}}^{(1)}\left(\bar{u}_{n} \mid \bar{\xi}_{\mathrm{I}}+c\right) \bar{K}_{n-1, l_{\mathrm{II}}}^{(1)}\left(\bar{u}_{n} \mid \bar{\xi}_{\mathrm{II}}-c\right) \nu_{12}\left(\bar{\xi}_{\mathrm{II}}\right)|0\rangle .
\end{aligned}
$$

Here $\bar{\xi}=\left\{\bar{u}_{n}, \bar{v}\right\}, l_{\mathrm{I}}=\# \bar{\xi}_{\mathrm{I}}$, and $l_{\mathrm{I}}=\# \bar{\xi}_{\mathrm{II}}$. The action of $\nu_{21}\left(u_{n}\right)$ gives us an additional sum over partitions

$$
\begin{aligned}
& \nu_{21}(\bar{u}) \nu_{12}(\bar{v})|0\rangle= \sum_{\bar{\xi} \Rightarrow\left\{\bar{\xi}_{\mathrm{I}}, \bar{\xi}_{\mathrm{I}}, \bar{\xi}_{\mathrm{II}}\right\}}\left(-\beta_{1}\right)^{n-l_{\mathrm{I}}}\left(-\beta_{2}\right)^{n-l_{\mathrm{II}}} \lambda_{2}\left(\bar{\xi}_{\mathrm{I}}\right) \lambda_{1}\left(\bar{\xi}_{\mathrm{II}}\right) f\left(\bar{\xi}_{\mathrm{I}}, \bar{\xi}_{\mathrm{II}}\right) f\left(\bar{\xi}_{\mathrm{I}}, \bar{\xi}_{\mathrm{II}}\right) f\left(\bar{\xi}_{\mathrm{II}}, \bar{\xi}_{\mathrm{II}}\right) \\
& \times K_{n-1, l_{\mathrm{I}}}^{(1)}\left(\bar{u}_{n} \mid \bar{\xi}_{\mathrm{I}}+c\right) \bar{K}_{n-1, l_{\mathrm{II}}}^{(1)}\left(\bar{u}_{n} \mid \bar{\xi}_{\mathrm{II}}-c\right) \sum_{\bar{\eta} \Rightarrow\left\{\bar{\eta}_{\mathrm{I}}, \bar{\eta}_{\mathrm{II}}, \bar{\eta}_{\mathrm{II}}\right\}}\left(-\beta_{1}\right)^{-k_{\mathrm{I}}}\left(-\beta_{2}\right)^{-k_{\mathrm{II}}} \lambda_{2}\left(\bar{\eta}_{\mathrm{I}}\right) \lambda_{1}\left(\bar{\eta}_{\mathrm{II}}\right) \\
& \times f\left(\bar{\eta}_{\mathrm{I}}, \bar{\eta}_{\mathrm{II}}\right) f\left(\bar{\eta}_{\mathrm{I}}, \bar{\eta}_{\mathrm{II}}\right) f\left(\bar{\eta}_{\mathrm{II}}, \bar{\eta}_{\mathrm{II}}\right) K_{1, k_{\mathrm{I}}}^{(1)}\left(u_{n} \mid \bar{\eta}_{\mathrm{I}}+c\right) \bar{K}_{1, k_{\mathrm{II}}}^{(1)}\left(u_{n} \mid \bar{\eta}_{\mathrm{II}}-c\right) \nu_{12}\left(\bar{\eta}_{\mathrm{III}}\right)|0\rangle,
\end{aligned}
$$

where $\bar{\eta}=\left\{\bar{\xi}_{\mathrm{II}}, v_{n}\right\}, k_{\mathrm{I}}=\# \bar{\eta}_{\mathrm{I}}$, and $k_{\mathrm{II}}=\# \bar{\eta}_{\mathrm{I}}$. Thus, eventually the sum is taken over partitions of the set $\{\bar{u}, \bar{v}\}$ into five subsets $\bar{\xi}_{\mathrm{I}}, \bar{\xi}_{\mathrm{II}}, \bar{\eta}_{\mathrm{I}}, \bar{\eta}_{\mathrm{II}}$, and $\bar{\eta}_{\mathrm{II}}$ such that $u_{n} \notin\left\{\bar{\xi}_{\mathrm{I}}, \bar{\xi}_{\mathrm{II}}\right\}$. The subset $\bar{\xi}_{\mathrm{II}}$ should be understood as $\bar{\xi}_{\mathrm{II}}=\left\{\bar{\eta}_{\mathrm{I}}, \bar{\eta}_{\mathrm{I}}, \bar{\eta}_{\mathrm{II}}\right\} \backslash\left\{u_{n}\right\}$.

Let $\bar{w}=\{\bar{u}, \bar{v}\}$. We denote $\bar{\xi}_{\mathrm{I}}=\bar{w}_{\mathrm{I}}, \bar{\xi}_{\mathrm{II}}=\bar{w}_{\mathrm{II}}, \bar{\eta}_{\mathrm{I}}=\bar{w}_{\mathrm{i}}, \bar{\eta}_{\mathrm{ii}}=\bar{w}_{\mathrm{ii}}$, and $\bar{\eta}_{\mathrm{II}}=\bar{w}_{\mathrm{iii}}$. Respectively, the cardinalities of the subsets are denoted by $r_{\mathrm{I}}=\# \bar{w}_{\mathrm{I}}, r_{\mathrm{II}}=\# \bar{w}_{\mathrm{II}}, r_{\mathrm{i}}=\# \bar{w}_{\mathrm{i}}, r_{\mathrm{ii}}=\# \bar{w}_{\mathrm{ii}}$. Then equation (5.28) takes the form

$$
\begin{aligned}
& \nu_{21}(\bar{u}) \nu_{12}(\bar{v})|0\rangle=\sum_{\bar{w} \Rightarrow\left\{\bar{w}_{\mathrm{I}}, \bar{w}_{\mathrm{II}}, \bar{w}_{\mathrm{i}}, \bar{w}_{\mathrm{ii}}, \bar{w}_{\mathrm{iii}}\right\}}\left(-\beta_{1}\right)^{n-r_{\mathrm{I}}-r_{\mathrm{i}}}\left(-\beta_{2}\right)^{n-r_{\mathrm{II}}-r_{\mathrm{ii}}} \lambda_{2}\left(\bar{w}_{\mathrm{i}}\right) \lambda_{2}\left(\bar{w}_{\mathrm{I}}\right) \lambda_{1}\left(\bar{w}_{\mathrm{II}}\right) \lambda_{1}\left(\bar{w}_{\mathrm{ii}}\right) \\
& \times \frac{f\left(\bar{w}_{\mathrm{I}}, \bar{w}_{\mathrm{II}}\right) f\left(\bar{w}_{\mathrm{I}}, \bar{w}_{\mathrm{i}}\right) f\left(\bar{w}_{\mathrm{I}}, \bar{w}_{\mathrm{ii}}\right) f\left(\bar{w}_{\mathrm{I}}, \bar{w}_{\mathrm{iii}}\right) f\left(\bar{w}_{\mathrm{i}}, \bar{w}_{\mathrm{II}}\right) f\left(\bar{w}_{\mathrm{ii}}, \bar{w}_{\mathrm{II}}\right) f\left(\bar{w}_{\mathrm{iii}}, \bar{w}_{\mathrm{II}}\right)}{f\left(\bar{w}_{\mathrm{I}}, u_{n}\right) f\left(u_{n}, \bar{w}_{\mathrm{II}}\right)} f\left(\bar{w}_{\mathrm{i}}, \bar{w}_{\mathrm{ii}}\right) f\left(\bar{w}_{\mathrm{i}}, \bar{w}_{\mathrm{iii}}\right) f\left(\bar{w}_{\mathrm{iii}}, \bar{w}_{\mathrm{ii}}\right) \\
& \times K_{n-1, r_{\mathrm{I}}}^{(1)}\left(\bar{u}_{n} \mid \bar{w}_{\mathrm{I}}+c\right) K_{1, r_{\mathrm{i}}}^{(1)}\left(u_{n} \mid \bar{w}_{\mathrm{i}}+c\right) \bar{K}_{n-1, r_{\mathrm{II}}}^{(1)}\left(\bar{u}_{n} \mid \bar{w}_{\mathrm{II}}-c\right) \bar{K}_{1, r_{\mathrm{ii}}}^{(1)}\left(u_{n} \mid \bar{w}_{\mathrm{ii}}-c\right) \nu_{12}\left(\bar{w}_{\mathrm{iii}}\right)|0\rangle . \quad(5.29)
\end{aligned}
$$


Observe that the restriction $u_{n} \notin\left\{\bar{w}_{\text {I }}, \bar{w}_{\text {II }}\right\}$ holds automatically, because $\left(f\left(\bar{w}_{\mathrm{I}}, u_{n}\right) f\left(u_{n}, \bar{w}_{\text {II }}\right)\right)^{-1}=0$ for $u_{n} \in\left\{\bar{w}_{\mathrm{I}}, \bar{w}_{\mathrm{II}}\right\}$. Setting $\left\{\bar{w}_{\mathrm{II}}, \bar{w}_{\mathrm{ii}}\right\}=\bar{w}_{0},\left\{\bar{w}_{\mathrm{I}}, \bar{w}_{\mathrm{i}}\right\}=\bar{w}_{0^{\prime}}$, and $\bar{w}_{\mathrm{iii}}=\bar{w}_{\mathrm{III}}$ we recast (5.29) as follows:

$$
\begin{aligned}
\nu_{21}(\bar{u}) \nu_{12}(\bar{v})|0\rangle=\sum_{\bar{w}=\left\{\bar{w}_{0^{\prime}}, \bar{w}_{0}, \bar{w}_{\mathrm{III}}\right\}}\left(-\beta_{1}\right)^{n-r_{0^{\prime}}}\left(-\beta_{2}\right)^{n-r_{0}} \lambda_{2}\left(\bar{w}_{0^{\prime}}\right) \lambda_{1}\left(\bar{w}_{0}\right) \nu_{12}\left(\bar{w}_{\text {III }}\right)|0\rangle \\
\quad \times f\left(\bar{w}_{0^{\prime}}, \bar{w}_{0}\right) f\left(\bar{w}_{0^{\prime}}, \bar{w}_{\text {III }}\right) f\left(\bar{w}_{\text {III }}, \bar{w}_{0}\right) W \bar{W},
\end{aligned}
$$

where $r_{0^{\prime}}=\# \bar{w}_{0^{\prime}}, r_{0}=\# \bar{w}_{0}$, and

$$
\begin{gathered}
W=\sum_{\bar{w}_{0^{\prime}} \Rightarrow\left\{\bar{w}_{\mathrm{I}}, \bar{w}_{\mathrm{i}}\right\}} \frac{f\left(\bar{w}_{\mathrm{I}}, \bar{w}_{\mathrm{i}}\right)}{f\left(\bar{w}_{\mathrm{I}}, u_{n}\right)} K_{n-1, r_{\mathrm{I}}}^{(1)}\left(\bar{u}_{n} \mid \bar{w}_{\mathrm{I}}+c\right) K_{1, r_{\mathrm{i}}}^{(1)}\left(u_{n} \mid \bar{w}_{\mathrm{i}}+c\right), \\
\bar{W}=\sum_{\bar{w}_{0} \Rightarrow\left\{\bar{w}_{\mathrm{II}}, \bar{w}_{\mathrm{ii}}\right\}} \frac{f\left(\bar{w}_{\mathrm{ii}}, \bar{w}_{\mathrm{II}}\right)}{f\left(u_{n}, \bar{w}_{\mathrm{II}}\right)} \bar{K}_{n-1, r_{\mathrm{II}}}^{(1)}\left(\bar{u}_{n} \mid \bar{w}_{\mathrm{II}}-c\right) \bar{K}_{1, r_{\mathrm{ii}}}^{(1)}\left(u_{n} \mid \bar{w}_{\mathrm{ii}}-c\right) .
\end{gathered}
$$

Observe that the sums over partitions of the subsets $\bar{w}_{0^{\prime}}$ and $\bar{w}_{0}$ can be obtained one from another via the replacement $c \rightarrow-c$. Moreover, the sum (5.32) was computed in (5.20). Thus,

$$
W=K_{n, r_{0^{\prime}}}^{(1)}\left(\bar{u} \mid \bar{w}_{0^{\prime}}+c\right), \quad \bar{W}=\bar{K}_{n, r_{0}}^{(1)}\left(\bar{u} \mid \bar{w}_{0}-c\right) .
$$

Substituting this into (5.30) we obtain

$$
\begin{aligned}
\nu_{21}(\bar{u}) \nu_{12}(\bar{v})|0\rangle= & \sum_{\bar{w}=\left\{\bar{w}_{0^{\prime}}, \bar{w}_{0}, \bar{w}_{\mathrm{II}}\right\}}\left(-\beta_{1}\right)^{n-r_{0^{\prime}}}\left(-\beta_{2}\right)^{n-r_{0}} \lambda_{2}\left(\bar{w}_{0^{\prime}}\right) \lambda_{1}\left(\bar{w}_{0}\right) \nu_{12}\left(\bar{w}_{\mathrm{II}}\right)|0\rangle \\
& \quad \times f\left(\bar{w}_{0^{\prime}}, \bar{w}_{0}\right) f\left(\bar{w}_{0^{\prime}}, \bar{w}_{\mathrm{III}}\right) f\left(\bar{w}_{\mathrm{III}}, \bar{w}_{0}\right) K_{n, r_{0^{\prime}}}^{(1)}\left(\bar{u} \mid \bar{w}_{0^{\prime}}+c\right) \bar{K}_{n, r_{0}}^{(1)}\left(\bar{u}^{\prime} \mid \bar{w}_{0}-c\right),
\end{aligned}
$$

which coincides with (5.22) up to the labels of the subsets. This ends the proof.

\subsection{Multiple action of the modified operator $\nu_{12}$}

Up to now all the multiple action formulas were valid for an arbitrary highest wight representation of the Yangian of $\mathfrak{g l}_{2}$. The following proposition is valid for finite dimensional representations only.

Proposition 5.3. Let $\# u=n$ and $\# v=m$. Consider an irreducible finite dimensional representation of the Yangian. Then there exists an integer $S$ and a function $F(u)$ such that for all $n$ and $m$ such that $m+n \geq S$ the following multiple action holds:

$$
\begin{aligned}
\nu_{12}(\bar{u}) \nu_{12}(\bar{v})|0\rangle=\nu_{12}(\bar{w})|0\rangle=\left(\frac{(\mu-1)\left(\beta_{1}+\beta_{2}\right)}{\beta_{1} \beta_{2}}\right)^{m+n-S} & \\
& \times \sum_{\bar{w} \Rightarrow\left\{\bar{w}_{\mathrm{I}}, \bar{w}_{\text {II }}\right\}} F\left(\bar{w}_{\mathrm{I}}\right) g\left(\bar{w}_{\mathrm{I}}, \bar{w}_{\text {II }}\right) \nu_{12}\left(\bar{w}_{\text {II }}\right)|0\rangle .
\end{aligned}
$$

The sum is taken over partitions $\{\bar{v}, \bar{u}\}=\bar{w} \Rightarrow\left\{\bar{w}_{\mathrm{I}}, \bar{w}_{\mathrm{II}}\right\}$ such that $\# \bar{w}_{\mathrm{I}}=m+n-S, \# \bar{w}_{\mathrm{II}}=S$. The constant $\mu$ is defined in (5.1). 
Remark 5.2. The value of $S$ and the explicit form of the function $F(u)$ depend on the concrete representation [2]. In particular, for the case of the fundamental representation of the inhomogeneous $X X X$ spin-1/2 chain with $N$ sites one has $S=N$ and

$$
F(u)=\prod_{i=1}^{N} \frac{h\left(u, \theta_{i}\right)}{g\left(u, \theta_{i}\right)}
$$

where $\theta_{i}$ are inhomogeneity parameters.

Remark 5.3. Equation (5.35) shows that if the number of the operators $\nu_{12}$ exceeds $S$, then their successive action on $|0\rangle$ reduces to the action of exactly $S$ such operators. This property is a peculiarity of finite-dimensional representations, and it is this property that is key for implementation of the MABA. In particular, the function $F(u)$ gives rise to the inhomogeneous term introduced in the context of the off-diagonal Bethe ansatz [41, 42]].

Proof. To prove proposition 5.3 we use induction over $n=\# \bar{u}$ with $n+m \geq S$. The case $n=1$ was first conjectured in [1] for the fundamental representation. Then, it was proved in [2] that for any irreducible finite dimension representation there exists an integer $S$ and a function $F(u)$ such that

$$
\begin{aligned}
\nu_{12}(u) \nu_{12}(\bar{v})=\frac{(\mu-1)\left(\beta_{1}+\beta_{2}\right)}{\beta_{1} \beta_{2}}\left(F(u) g(u, \bar{v}) \nu_{12}\right. & (\bar{v}) \\
& \left.+\sum_{i=1}^{S} g\left(v_{i}, u\right) F\left(v_{i}\right) g\left(v_{i}, \bar{v}_{i}\right) \nu_{12}(u) \nu_{12}\left(\bar{v}_{i}\right)\right) .
\end{aligned}
$$

The reader can find the explicit form of $F(u)$ and the corresponding $S$ in [2]. It is easy to see that the term in the first line of (5.37) corresponds to the partition $\bar{w}_{\mathrm{I}}=u, \bar{w}_{\mathrm{II}}=\bar{v}$ in (5.35). The terms in the second line of (5.37) correspond to the partitions $\bar{w}_{\mathrm{I}}=v_{i}, \bar{w}_{\mathrm{II}}=\left\{u, \bar{v}_{i}\right\}(i=1, \ldots, m)$ in (5.35). Thus, (5.35) coincides with (5.37) for $n=1$.

Let (5.35) be valid for $n-1=\# \bar{u}_{n}$ such that $n-1+m>S$. Consider the action of $\nu_{12}(\bar{u})$ with $n=\# \bar{u}$. We can act successively, firstly by $\nu_{12}\left(\bar{u}_{n}\right)$ and secondly by $\nu_{12}\left(u_{n}\right)$. Due to the induction assumption we obtain at the first step

$$
\nu_{12}(\bar{u}) \nu_{12}(\bar{v})|0\rangle=\left(\frac{(\mu-1)\left(\beta_{1}+\beta_{2}\right)}{\beta_{1} \beta_{2}}\right)^{m+n-S-1} \sum_{\bar{\xi} \Rightarrow\left\{\bar{\xi}_{\mathrm{I}}, \bar{\xi}_{\mathrm{II}}\right\}} F\left(\bar{\xi}_{\mathrm{I}}\right) g\left(\bar{\xi}_{\mathrm{I}}, \bar{\xi}_{\mathrm{II}}\right) \nu_{12}\left(u_{n}\right) \nu_{12}\left(\bar{\xi}_{\mathrm{II}}\right)|0\rangle,
$$

where the sum is taken over partitions $\bar{\xi}=\left\{\bar{u}_{n}, \bar{v}\right\} \Rightarrow\left\{\bar{\xi}_{\mathrm{I}}, \bar{\xi}_{\mathrm{I}}\right\}$ such that $\# \bar{\xi}_{\mathrm{I}}=n-1, \# \bar{\xi}_{\mathrm{II}}=m$. Acting with $\nu_{12}\left(u_{n}\right)$ on $\nu_{12}\left(\bar{\xi}_{\text {II }}\right)|0\rangle$ via (5.37) we find

$$
\begin{aligned}
& \nu_{12}(\bar{u}) \nu_{12}(\bar{v})|0\rangle=\left(\frac{(\mu-1)\left(\beta_{1}+\beta_{2}\right)}{\beta_{1} \beta_{2}}\right)^{m+n-S} \\
& \quad \times \sum_{\bar{\xi} \Leftrightarrow\left\{\bar{\xi}_{\mathrm{I}}, \bar{\xi}_{\mathrm{I}}\right\}} \sum_{\bar{\eta} \Rightarrow\left\{\bar{\eta}_{\mathrm{I}}, \bar{\eta}_{\mathrm{II}}\right\}} F\left(\bar{\xi}_{\mathrm{I}}\right) F\left(\bar{\eta}_{\mathrm{I}}\right) g\left(\bar{\xi}_{\mathrm{I}}, \bar{\xi}_{\mathrm{II}}\right) g\left(\bar{\eta}_{\mathrm{I}}, \bar{\eta}_{\mathrm{II}}\right) \nu_{12}\left(\bar{\eta}_{\mathrm{II}}\right)|0\rangle,
\end{aligned}
$$


where we have additional partitions $\bar{\eta}=\left\{u_{n}, \bar{\xi}_{\mathrm{II}}\right\} \Rightarrow\left\{\bar{\eta}_{\mathrm{I}}, \bar{\eta}_{\mathrm{II}}\right\}$ such that $\# \bar{\eta}_{\mathrm{I}}=1$ and $\# \bar{\eta}_{\mathrm{II}}=m$. Thus, eventually we deal with the partitions of the set $\bar{w}=\{\bar{u}, \bar{v}\}$ into three subsets: $\bar{\xi}_{\mathrm{I}}$, $\bar{\eta}_{\mathrm{I}}$, and $\bar{\eta}_{\mathrm{I}}$. The subset $\bar{\xi}_{\mathrm{II}}$ should be understood as $\bar{\xi}_{\mathrm{II}}=\left\{\bar{\eta}_{\mathrm{I}}, \bar{\eta}_{\mathrm{II}}\right\} \backslash\left\{u_{n}\right\}$. Besides the restrictions on the cardinalities of the subsets we have the additional restriction $u_{n} \notin \bar{\xi}_{\mathrm{I}}$.

Let $\bar{\xi}_{\mathrm{I}}=\bar{w}_{\mathrm{I}}, \bar{\eta}_{\mathrm{I}}=\bar{w}_{\mathrm{II}}$, and $\bar{\eta}_{\mathrm{II}}=\bar{w}_{\mathrm{III}}$. Then $\bar{\xi}_{\mathrm{II}}=\left\{\bar{w}_{\mathrm{II}}, \bar{w}_{\mathrm{III}}\right\} \backslash\left\{u_{n}\right\}$, and equation (5.39) takes the form

$$
\begin{aligned}
& \nu_{12}(\bar{u}) \nu_{12}(\bar{v})|0\rangle=\left(\frac{(\mu-1)\left(\beta_{1}+\beta_{2}\right)}{\beta_{1} \beta_{2}}\right)^{m+n-S} \sum_{\bar{w} \Rightarrow\left\{\bar{w}_{\mathrm{I}}, \bar{w}_{\mathrm{II}}, \bar{w}_{\mathrm{III}}\right\}} F\left(\bar{w}_{\mathrm{I}}\right) F\left(\bar{w}_{\mathrm{II}}\right) \nu_{12}\left(\bar{w}_{\mathrm{III}}\right)|0\rangle \\
& \times \frac{g\left(\bar{w}_{\mathrm{I}}, \bar{w}_{\mathrm{II}}\right) g\left(\bar{w}_{\mathrm{I}}, \bar{w}_{\mathrm{III}}\right)}{g\left(\bar{w}_{\mathrm{I}}, u_{n}\right)} g\left(\bar{w}_{\mathrm{II}}, \bar{w}_{\mathrm{III}}\right) .
\end{aligned}
$$

Observe that the condition $u_{n} \notin \bar{w}_{\mathrm{I}}$ is valid automatically due to the factor $\left(g\left(\bar{w}_{\mathrm{I}}, u_{n}\right)\right)^{-1}$ that vanishes if $u_{n} \in \bar{w}_{\mathrm{I}}$. Setting $\bar{w}_{0}=\left\{\bar{w}_{\mathrm{I}}, \bar{w}_{\mathrm{II}}\right\}$ we recast (5.40) as follows:

$$
\begin{aligned}
\nu_{12}(\bar{u}) \nu_{12}(\bar{v})|0\rangle=\left(\frac{(\mu-1)\left(\beta_{1}+\beta_{2}\right)}{\beta_{1} \beta_{2}}\right)^{m+n-S} \sum_{\bar{w} \Rightarrow\left\{\bar{w}_{0}, \bar{w}_{\text {III }}\right.} F\left(\bar{w}_{0}\right) g\left(\bar{w}_{0}, \bar{w}_{\text {III }}\right) \nu_{12}\left(\bar{w}_{\text {III }}\right)|0\rangle \\
\times \sum_{\bar{w}_{0} \Rightarrow\left\{\bar{w}_{\mathrm{I}}, \bar{w}_{\text {II }}\right\}} \frac{g\left(\bar{w}_{\mathrm{I}}, \bar{w}_{\text {II }}\right)}{g\left(\bar{w}_{\mathrm{I}}, u_{n}\right)} .
\end{aligned}
$$

The sum over partitions is now taken in two steps. First, the set $\bar{w}=\{\bar{u}, \bar{v}\}$ is divided into subsets $\left\{\bar{w}_{0}, \bar{w}_{\text {III }}\right\}$ such that $\# \bar{w}_{0}=n$ and $\# \bar{w}_{\text {III }}=m$. Then the subset $\bar{w}_{0}$ is divided into subsets $\left\{\bar{w}_{\mathrm{I}}, \bar{w}_{\text {II }}\right\}$ such that $\# \bar{w}_{\mathrm{I}}=n-1$ and $\# \bar{w}_{\mathrm{II}}=1$. Let us prove that the latter sum is equal to 1 . We have

$$
\sum_{\bar{w}_{0} \Rightarrow\left\{\bar{w}_{\mathrm{I}}, \bar{w}_{\text {II }}\right.} \frac{g\left(\bar{w}_{\mathrm{I}}, \bar{w}_{\text {II }}\right)}{g\left(\bar{w}_{\mathrm{I}}, u_{n}\right)}=\lim _{x \rightarrow u_{n}} \frac{1}{g\left(\bar{w}_{0}, x\right)} \sum_{\bar{w}_{0} \Rightarrow\left\{\bar{w}_{\mathrm{I}}, \bar{w}_{\text {II }}\right\}} g\left(\bar{w}_{\mathrm{I}}, \bar{w}_{\text {II }}\right) g\left(\bar{w}_{\text {II }}, x\right) .
$$

Here we have replaced $u_{n}$ by $x$ in order to avoid possible singularity at $\bar{w}_{\text {II }}=u_{n}$. Recall that $\# \bar{w}_{\text {II }}=1$. Thus, the sum over partitions in the right hand side of (5.42) is given by a contour integral

$$
\sum_{\bar{w}_{0} \Rightarrow\left\{\bar{w}_{\mathrm{I}}, \bar{w}_{\mathrm{II}}\right\}} g\left(\bar{w}_{\mathrm{I}}, \bar{w}_{\mathrm{II}}\right) g\left(\bar{w}_{\mathrm{II}}, x\right)=\frac{-1}{2 \pi i c} \oint_{\Gamma\left(\bar{w}_{0}\right)} g\left(\bar{w}_{0}, z\right) g(z, x) d z
$$

where anticlockwise oriented contour $\Gamma\left(\bar{w}_{0}\right)$ surrounds the points $\bar{w}_{0}$ and does not contain any other singularities of the integrand. Taking the integral by the residue outside the integration contour (that is, at $z=x$ ) we immediately obtain

$$
\sum_{\bar{w}_{0} \Rightarrow\left\{\bar{w}_{\mathrm{I}}, \bar{w}_{\text {II }}\right\}} g\left(\bar{w}_{\mathrm{I}}, \bar{w}_{\mathrm{II}}\right) g\left(\bar{w}_{\mathrm{II}}, x\right)=g\left(\bar{w}_{0}, x\right)
$$

leading to

$$
\sum_{\bar{w}_{0} \Rightarrow\left\{\bar{w}_{\mathrm{I}}, \bar{w}_{\text {II }}\right\}} \frac{g\left(\bar{w}_{\mathrm{I}}, \bar{w}_{\text {II }}\right)}{g\left(\bar{w}_{\mathrm{I}}, u_{n}\right)}=1 .
$$


Substituting this into (5.41) we arrive at

$$
\nu_{12}(\bar{u}) \nu_{12}(\bar{v})|0\rangle=\left(\frac{(\mu-1)\left(\beta_{1}+\beta_{2}\right)}{\beta_{1} \beta_{2}}\right)^{m+n-S} \sum_{\bar{w} \Rightarrow\left\{\bar{w}_{0}, \bar{w}_{\text {III }}\right.} F\left(\bar{w}_{0}\right) g\left(\bar{w}_{0}, \bar{w}_{\text {III }}\right) \nu_{12}\left(\bar{w}_{\text {III }}\right)|0\rangle,
$$

which coincides with (5.35) up to the labels of the subsets. Thus, the proof is completed.

\section{Modified scalar product}

We can now consider the scalar product of the modified Bethe vectors.

Theorem 6.1. Let $\# \bar{u}=n$ and $\# \bar{v}=m$. Then the scalar product of two modified Bethe vectors

$$
S_{\nu}^{n, m}(\bar{u}, \bar{v})=\left\langle 0\left|\nu_{21}(\bar{u}) \nu_{12}(\bar{v})\right| 0\right\rangle
$$

is given by

$$
S_{\nu}^{n, m}(\bar{u}, \bar{v})=\sum_{\bar{\xi} \Rightarrow\left\{\bar{\xi}_{\mathrm{I}}, \bar{\xi}_{\Pi}\right\}}\left(-\beta_{1}\right)^{n-l_{\mathrm{I}}}\left(-\beta_{2}\right)^{n-l_{\mathrm{II}}} \lambda_{2}\left(\bar{\xi}_{\mathrm{I}}\right) \lambda_{1}\left(\bar{\xi}_{\mathrm{II}}\right) f\left(\bar{\xi}_{\mathrm{I}}, \bar{\xi}_{\mathrm{II}}\right) K_{n, l_{\mathrm{I}}}^{(\mu)}\left(\bar{u} \mid \bar{\xi}_{\mathrm{I}}+c\right) \bar{K}_{n, l_{\mathrm{II}}}^{(\mu)}\left(\bar{u} \mid \bar{\xi}_{\mathrm{II}}-c\right) .
$$

Here $\bar{\xi}=\{\bar{u}, \bar{v}\}, l_{\mathrm{I}}=\bar{\xi}_{\mathrm{I}}$, and $l_{\mathrm{II}}=\bar{\xi}_{\mathrm{I}}$. The sum is taken over all partitions $\bar{\xi} \Rightarrow\left\{\bar{\xi}_{\mathrm{I}}, \bar{\xi}_{\mathrm{I}}\right\}$. There is no restriction on the cardinalities of the subsets. The functions $K_{n, l_{\mathrm{I}}}^{(\mu)}$ and $\bar{K}_{n, l_{\mathrm{II}}}^{(\mu)}$ respectively are the modified Izergin determinants (3.5) and (3.7) at $z=\mu$.

Proof. Acting with the dual highest weight vector (2.8) onto (5.22) we find

$$
\begin{aligned}
S_{\nu}^{n, m}(\bar{u}, \bar{v})=\sum_{\bar{\xi} \Rightarrow\left\{\bar{\xi}_{\mathrm{I}}, \bar{\xi}_{\mathrm{II}}, \bar{\xi}_{\mathrm{II}}\right\}}\left(-\beta_{1}\right)^{n-l_{\mathrm{I}}}\left(-\beta_{2}\right)^{n-l_{\mathrm{II}}} & \lambda_{2}\left(\bar{\xi}_{\mathrm{I}}\right) \lambda_{1}\left(\bar{\xi}_{\mathrm{II}}\right) f\left(\bar{\xi}_{\mathrm{I}}, \bar{\xi}_{\mathrm{II}}\right) f\left(\bar{\xi}_{\mathrm{I}}, \bar{\xi}_{\mathrm{III}}\right) f\left(\bar{\xi}_{\mathrm{II}}, \bar{\xi}_{\mathrm{II}}\right) \\
& \times K_{n, l_{\mathrm{I}}}^{(1)}\left(\bar{u} \mid \bar{\xi}_{\mathrm{I}}+c\right) \bar{K}_{n, l_{\mathrm{II}}}^{(1)}\left(\bar{u} \mid \bar{\xi}_{\mathrm{II}}-c\right)\left\langle 0\left|\nu_{12}\left(\bar{\xi}_{\mathrm{II}}\right)\right| 0\right\rangle .
\end{aligned}
$$

Recall that here $\bar{\xi}=\{\bar{u}, \bar{v}\}, \# \bar{\xi}_{\mathrm{I}}=l_{\mathrm{I}}$, and $\# \bar{\xi}_{\mathrm{II}}=l_{\mathrm{II}}$. The sum is taken over all partitions $\bar{\xi} \Rightarrow$ $\left\{\bar{\xi}_{\mathrm{I}}, \bar{\xi}_{\mathrm{II}}, \bar{\xi}_{\mathrm{III}}\right\}$.

The vacuum average $\left\langle 0\left|\nu\left(\bar{\xi}_{\mathrm{II}}\right)\right| 0\right\rangle$ was computed in 35$]$ :

$$
\left\langle 0\left|\nu_{12}(\bar{w})\right| 0\right\rangle=(1-\mu)^{p} \sum_{\bar{w} \Rightarrow\left\{\bar{w}_{\mathrm{I}}, \bar{w}_{\mathrm{II}}\right\}}\left(-\beta_{2}\right)^{-\# \bar{w}_{\mathrm{II}}}\left(-\beta_{1}\right)^{-\# \bar{w}_{\mathrm{I}}} \lambda_{2}\left(\bar{w}_{\mathrm{I}}\right) \lambda_{1}\left(\bar{w}_{\mathrm{II}}\right) f\left(\bar{w}_{\mathrm{I}}, \bar{w}_{\mathrm{II}}\right),
$$

where $\# \bar{w}=p$ and the sum is taken over all partitions $\bar{w} \Rightarrow\left\{\bar{w}_{\mathrm{I}}, \bar{w}_{\mathrm{II}}\right\}$. Substituting (6.4) into (6.3) and decomposing $\bar{\xi}_{\text {III }}=\left\{\bar{\xi}_{\text {i }}, \bar{\xi}_{\text {ii }}\right\}$ we find

$$
\begin{aligned}
S_{\nu}^{n, m}(\bar{u}, \bar{v}) & =\sum_{\bar{\xi} \Rightarrow\left\{\bar{\xi}_{\mathrm{I}}, \bar{\xi}_{\mathrm{II}}, \bar{\xi}_{\mathrm{i}}, \bar{\xi}_{\mathrm{ii}}\right\}}(1-\mu)^{l_{\mathrm{i}}+l_{\mathrm{ii}}}\left(-\beta_{1}\right)^{n-l_{\mathrm{I}}-l_{\mathrm{i}}}\left(-\beta_{2}\right)^{n-l_{\mathrm{II}}-l_{\mathrm{ii}}} \lambda_{2}\left(\bar{\xi}_{\mathrm{I}}\right) \lambda_{1}\left(\bar{\xi}_{\mathrm{II}}\right) \lambda_{2}\left(\bar{\xi}_{\mathrm{i}}\right) \lambda_{1}\left(\bar{\xi}_{\mathrm{ii}}\right) \\
& \times f\left(\bar{\xi}_{\mathrm{I}}, \bar{\xi}_{\mathrm{II}}\right) f\left(\bar{\xi}_{\mathrm{I}}, \bar{\xi}_{\mathrm{i}}\right) f\left(\bar{\xi}_{\mathrm{I}}, \bar{\xi}_{\mathrm{ii}}\right) f\left(\bar{\xi}_{\mathrm{i}}, \bar{\xi}_{\mathrm{II}}\right) f\left(\bar{\xi}_{\mathrm{ii}}, \bar{\xi}_{\mathrm{II}}\right) f\left(\bar{\xi}_{\mathrm{i}}, \bar{\xi}_{\mathrm{ii}}\right) K_{n, l_{\mathrm{I}}}^{(1)}\left(\bar{u} \mid \bar{\xi}_{\mathrm{I}}+c\right) \bar{K}_{n, l_{\mathrm{II}}}^{(1)}\left(\bar{u}_{\mid} \mid \bar{\xi}_{\mathrm{II}}-c\right) .
\end{aligned}
$$


Here the sum is taken over partitions $\bar{\xi} \Rightarrow\left\{\bar{\xi}_{\mathrm{I}}, \bar{\xi}_{\mathrm{II}}, \bar{\xi}_{\mathrm{i}}, \bar{\xi}_{\mathrm{ii}}\right\}$. The cardinalities of the subsets are denoted by $l$ with the corresponding subscript.

Now we set $\left\{\bar{\xi}_{\mathrm{I}}, \bar{\xi}_{\mathrm{i}}\right\}=\bar{\xi}_{0},\left\{\bar{\xi}_{\mathrm{II}}, \bar{\xi}_{\mathrm{ii}}\right\}=\bar{\xi}_{0^{\prime}}$. Then we arrive at

$$
S_{\nu}^{n, m}(\bar{u}, \bar{v})=\sum_{\bar{\xi} \Rightarrow\left\{\bar{\xi}_{0}, \bar{\xi}_{0^{\prime}}\right\}}\left(-\beta_{1}\right)^{n-l_{0}}\left(-\beta_{2}\right)^{n-l_{0^{\prime}}} \lambda_{2}\left(\bar{\xi}_{0}\right) \lambda_{1}\left(\bar{\xi}_{0^{\prime}}\right) f\left(\bar{\xi}_{0}, \bar{\xi}_{0^{\prime}}\right) \mathcal{L}\left(\bar{\xi}_{0}\right) \overline{\mathcal{L}}\left(\bar{\xi}_{0^{\prime}}\right),
$$

where

$$
\mathcal{L}\left(\bar{\xi}_{0}\right)=\sum_{\bar{\xi}_{0} \Rightarrow\left\{\bar{\xi}_{\mathrm{I}}, \bar{\xi}_{\mathrm{i}}\right\}}(1-\mu)^{l_{\mathrm{i}}} K_{n, l_{\mathrm{I}}}^{(1)}\left(\bar{u} \mid \bar{\xi}_{\mathrm{I}}+c\right) f\left(\bar{\xi}_{\mathrm{I}}, \bar{\xi}_{\mathrm{i}}\right)
$$

and

$$
\overline{\mathcal{L}}\left(\bar{\xi}_{0^{\prime}}\right)=\sum_{\bar{\xi}_{0^{\prime}} \Rightarrow\left\{\bar{\xi}_{\mathrm{II}}, \bar{\xi}_{\mathrm{ii}}\right\}}(1-\mu)^{l_{\mathrm{ii}}} \bar{K}_{n, l_{\mathrm{II}}}^{(1)}\left(\bar{u} \mid \bar{\xi}_{\mathrm{II}}-c\right) f\left(\bar{\xi}_{\mathrm{ii}}, \bar{\xi}_{\mathrm{II}}\right) .
$$

The sums (6.7) and (6.8) are computed in proposition A.9.

$$
\mathcal{L}\left(\bar{\xi}_{0}\right)=K_{n, l_{0}}^{(\mu)}\left(\bar{u} \mid \bar{\xi}_{0}+c\right), \quad \overline{\mathcal{L}}\left(\bar{\xi}_{0^{\prime}}\right)=\bar{K}_{n, l_{0^{\prime}}}^{(\mu)}\left(\bar{u} \mid \bar{\xi}_{0^{\prime}}-c\right) .
$$

Then equation (6.6) coincides with (6.2) up to the labels of the subsets.

Remarkably, this formula has exactly the same form as representation (4.7) for the scalar product in the usual ABA (for $m=n$ ). However, instead of the ordinary Izergin determinants we have now modified Izergin determinants. Furthermore, we have no restrictions on the cardinalities of the subsets.

Consider the case $\mu=1$ and $n=m$. Then, due to (3.10) a non-vanishing contribution occurs if and only if $n \geq \# \bar{\xi}_{\mathrm{I}}$ and $n \geq \# \bar{\xi}_{\mathrm{II}}$. Since $\# \bar{\xi}_{\mathrm{I}}+\# \bar{\xi}_{\mathrm{II}}=2 n$, we conclude that $n=\# \bar{\xi}_{\mathrm{I}}$ and $n=\# \bar{\xi}_{\mathrm{II}}$. This leads us to

$$
\left.S_{\nu}^{n, n}(\bar{u}, \bar{v})\right|_{\mu=1}=\sum_{\substack{\bar{\xi} \rightrightarrows\left\{\bar{\xi}_{\mathrm{I}}, \bar{\xi}_{\mathrm{II}}\right\} \\ \# \bar{\xi}_{\mathrm{I}}=\# \bar{\xi}_{\mathrm{II}}=n}} \lambda_{2}\left(\bar{\xi}_{\mathrm{I}}\right) \lambda_{1}\left(\bar{\xi}_{\mathrm{II}}\right) f\left(\bar{\xi}_{\mathrm{I}}, \bar{\xi}_{\mathrm{II}}\right) K_{n}\left(\bar{u} \mid \bar{\xi}_{\mathrm{I}}+c\right) \bar{K}_{n}\left(\bar{u} \mid \bar{\xi}_{\mathrm{II}}-c\right),
$$

and we reproduce the usual ABA scalar product $S_{t}^{n}$ given by theorem 4.1 .

Similarly to (4.8) the sum (6.2) can be written in the form of the sum over independent partitions of the sets $\bar{u}$ and $\bar{v}$ (modified Izergin-Korepin formula).

Corollary 6.1. Let $\# \bar{u}=n$ and $\# \bar{v}=m$. Then the modified scalar product of two Bethe vectors is given by

$$
\begin{aligned}
S_{\nu}^{n, m}(\bar{u}, \bar{v})=\mu^{2 n}(1-\mu)^{m-n} \sum\left(-\beta_{1}\right)^{n_{2}-m_{2}}\left(-\beta_{2}\right)^{n_{1}-m_{1}} \lambda_{2}\left(\bar{u}_{\mathrm{I}}\right) \lambda_{2}\left(\bar{v}_{\mathrm{II}}\right) \lambda_{1}\left(\bar{u}_{\mathrm{II}}\right) \lambda_{1}\left(\bar{v}_{\mathrm{I}}\right) \\
\times f\left(\bar{u}_{\mathrm{I}}, \bar{u}_{\mathrm{II}}\right) f\left(\bar{v}_{\mathrm{II}}, \bar{v}_{\mathrm{I}}\right) K_{m_{2}, n_{2}}^{(1 / \mu)}\left(\bar{v}_{\mathrm{II}} \mid \bar{u}_{\mathrm{II}}\right) \bar{K}_{m_{1}, n_{1}}^{(1 / \mu)}\left(\bar{v}_{\mathrm{I}} \mid \bar{u}_{\mathrm{I}}\right),
\end{aligned}
$$

where the sum is taken over all partitions $\bar{u} \Rightarrow\left\{\bar{u}_{\mathrm{I}}, \bar{u}_{\mathrm{II}}\right\}$ and $\bar{v} \Rightarrow\left\{\bar{v}_{\mathrm{I}}, \bar{v}_{\mathrm{II}}\right\}$ such that $\# \bar{v}_{\mathrm{I}}=m_{1}$, $\# \bar{v}_{\mathrm{II}}=m_{2}$ and $\# \bar{u}_{\mathrm{I}}=n_{1}, \# \bar{u}_{\mathrm{II}}=n_{2}$, where $n_{1}=0,1, \ldots, n$ and $m_{1}=0,1, \ldots, m$. 
Proof. We set $\bar{w}_{\mathrm{I}} \Rightarrow\left\{\bar{u}_{\mathrm{I}}, \bar{v}_{\mathrm{II}}\right\}$ and $\bar{w}_{\mathrm{II}} \Rightarrow\left\{\bar{u}_{\mathrm{II}}, \bar{v}_{\mathrm{I}}\right\}$ with $\# \bar{u}_{\mathrm{I}}=n_{1}, \# \bar{v}_{\mathrm{I}}=m_{1}, \# \bar{u}_{\mathrm{II}}=n_{2}, \# \bar{v}_{\mathrm{II}}=m_{2}$ and $n=n_{1}+n_{2}, m=m_{1}+m_{2}$ in (6.2). Using (A.5) and (A.6) we obtain:

$$
\begin{aligned}
S_{\nu}^{n, m}(\bar{u}, \bar{v})=(-\mu)^{n} & \sum_{\substack{\bar{u} \Rightarrow\left\{\bar{u}_{\mathrm{I}}, \bar{u}_{\mathrm{II}}\right\} \\
\bar{v} \Rightarrow\left\{\overline{\mathrm{I}}_{\mathrm{I}}, \overline{\mathrm{I}}_{\mathrm{II}}\right\}}}\left(-\beta_{1}\right)^{n-n_{1}-m_{2}}\left(-\beta_{2}\right)^{n-n_{2}-m_{1}} \lambda_{2}\left(\bar{u}_{\mathrm{I}}\right) \lambda_{2}\left(\bar{v}_{\mathrm{II}}\right) \lambda_{1}\left(\bar{u}_{\mathrm{II}}\right) \lambda_{1}\left(\bar{v}_{\mathrm{I}}\right) \\
\times & K_{n_{2}, m_{2}}^{(\mu)}\left(\bar{u}_{\mathrm{II}} \mid \bar{v}_{\mathrm{II}}+c\right) \bar{K}_{n_{1}, m_{1}}^{(\mu)}\left(\bar{u}_{\mathrm{I}} \mid \bar{v}_{\mathrm{I}}-c\right) f\left(\bar{u}_{\mathrm{I}}, \bar{u}_{\mathrm{II}}\right) f\left(\bar{v}_{\mathrm{II}}, \bar{v}_{\mathrm{I}}\right) f\left(\bar{u}_{\mathrm{I}}, \bar{v}_{\mathrm{I}}\right) f\left(\bar{v}_{\mathrm{II}}, \bar{u}_{\mathrm{II}}\right) .
\end{aligned}
$$

Then the use of (A.15) and (A.16) for the modified Izergin determinants immediately gives (6.11).

\section{Conclusion}

We considered multiple actions of the modified monodromy matrix entries on the modified Bethe vectors within the framework of the MABA. We shown that they look very similar to the standard multiple actions obtained for the ordinary ABA in [31]. The main difference is that the ordinary Izergin determinant [37] is modified according to (3.5) and (3.7), and the sum over partitions of the Bethe parameters should be taken without restrictions on the cardinalities of the subsets. The same changes apply to the formula for the scalar product of the modified Bethe vectors. It would be interesting to compare this result with those that follow from the separation of variable approach [43].

Further development of the method, as the one proposed in this paper, can be carried out in several directions. It is quite possible that the multiple action formulas admit a deformation to the XXZ model. In this case, however, the property (2.3) is no longer valid for arbitrary twist matrices. Therefore, one should consider a more sophisticated face-vertex transformation of the twist (see e.g. [45, 46] and references therein).

It is also interesting to consider models with higher rank algebra. The main open problem in this direction is to construct the Bethe vectors in the twisted periodic case.

Finally, a very attractive way for further development is to consider particular cases of the scalar products of the modified Bethe vectors. It is well known from the ABA that if one of the vectors is an eigenvector of the transfer matrix (on-shell Bethe vector), then the scalar product admit a compact determinant representation, which involves the Jacobian of the transfer matrix eigenvalue [5]. It was conjectured in [1] that a similar representation also exists in the case of the scalar products involving the modified on-shell Bethe vectors. We will provide a proof of this conjecture in our forthcoming publication.

\section{Acknowledgements}

N. S. would like to thank the hospitality of the Institute de Physique Théorique at the CEA de Saclay where a part of this work was done. S.B. and B.V. would like to thank the hospitality of the LMPT of Tours where a part of this work was done. S.B. was supported by a public grant as part of the Investissement d'avenir project, reference ANR-11-LABX-0056-LMH, LabEx LMH. N.S. was supported by the Russian Foundation RFBR-18-01-00273a. 


\section{A Properties of modified Izergin determinant}

In this section we give a list of properties of the modified Izergin determinant introduced in section 3.1. In all the propositions listed below $\bar{u}$ and $\bar{v}$ are two sets of arbitrary complex numbers with cardinalities $\# \bar{u}=n$ and $\# \bar{v}=m$.

\section{A.1 Basis properties}

\section{Proposition A.1.}

$$
\begin{gathered}
K_{n, m}^{(z)}(\bar{u}-c \mid \bar{v})=K_{n, m}^{(z)}(\bar{u} \mid \bar{v}+c), \\
\bar{K}_{n, m}^{(z)}(\bar{u}-c \mid \bar{v})=\bar{K}_{n, m}^{(z)}(\bar{u} \mid \bar{v}+c) . \\
K_{n, m}^{(z)}(-\bar{u} \mid-\bar{v})=\bar{K}_{n, m}^{(z)}(\bar{u} \mid \bar{v}) .
\end{gathered}
$$

Proof. These formulas directly follow from (3.5) - (3.8) and the definition of the rational functions (3.1).

\section{Proposition A.2.}

$$
\begin{aligned}
K_{n, 0}^{(z)}(\bar{u} \mid \emptyset)=\bar{K}_{n, 0}^{(z)}(\bar{u} \mid \emptyset)= & 1, \quad K_{0, n}^{(z)}(\emptyset \mid \bar{v})=\bar{K}_{0, n}^{(z)}(\emptyset \mid \bar{v})=(1-z)^{n}, \\
K_{1, m}^{(z)}(u \mid \bar{v}) & =(1-z)^{m-1}(f(u, \bar{v})-z), \\
K_{n, 1}^{(z)}(\bar{u} \mid v) & =f(\bar{u}, v)-z \\
\bar{K}_{1, m}^{(z)}(u \mid \bar{v}) & =(1-z)^{m-1}(f(\bar{v}, u)-z), \\
\bar{K}_{n, 1}^{(z)}(\bar{u} \mid v) & =f(v, \bar{u})-z .
\end{aligned}
$$

Proof. These formulas directly follow from (3.5)- (3.8).

Proposition A.3.

$$
\begin{aligned}
& K_{n+1, m+1}^{(z)}(\{\bar{u}, w-c\} \mid\{\bar{v}, w\})=-z K_{n, m}^{(z)}(\bar{u} \mid \bar{v}) . \\
& \bar{K}_{n+1, m+1}^{(z)}(\{\bar{u}, w+c\} \mid\{\bar{v}, w\})=-z \bar{K}_{n, m}^{(z)}(\bar{u} \mid \bar{v}) .
\end{aligned}
$$

Proof. We use representation (3.5). We see that only the term $-z \delta_{m+1, k}$ survives in the last row of the determinant due to $f(w-c, w)=0$. Then we obtain

$$
\begin{array}{r}
K_{n+1, m+1}^{(z)}(\{\bar{u}, w-c\} \mid\{\bar{v}, w\})=-z \operatorname{det}_{m}\left(-z \delta_{j k}+\frac{f\left(\bar{u}, v_{j}\right) f\left(w-c, v_{j}\right) f\left(v_{j}, \bar{v}_{j}\right) f\left(v_{j}, w\right)}{h\left(v_{j}, v_{k}\right)}\right) \\
=-z \operatorname{det}_{m}\left(-z \delta_{j k}+\frac{f\left(\bar{u}, v_{j}\right) f\left(v_{j}, \bar{v}_{j}\right)}{h\left(v_{j}, v_{k}\right)}\right)=-z K_{n, m}^{(z)}(\bar{u} \mid \bar{v}),
\end{array}
$$

because $f\left(w-c, v_{j}\right) f\left(v_{j}, w\right)=1$ due to (3.3). Equation (A.6) then follows from the replacement $c \rightarrow-c$. 


\section{Proposition A.4.}

$$
\begin{aligned}
& K_{n, m}^{(z)}(\bar{u} \mid \bar{v})=\sum_{\bar{v} \Rightarrow\left\{\bar{v}_{\mathrm{I}}, \bar{v}_{\mathrm{II}}\right\}}(-z)^{\# \bar{v}_{\mathrm{II}}} f\left(\bar{u}, \bar{v}_{\mathrm{I}}\right) f\left(\bar{v}_{\mathrm{I}}, \bar{v}_{\mathrm{II}}\right), \\
& \bar{K}_{n, m}^{(z)}(\bar{u} \mid \bar{v})=\sum_{\bar{v} \Rightarrow\left\{\bar{v}_{\mathrm{I}}, \bar{v}_{\mathrm{II}}\right\}}(-z)^{\# \bar{v}_{\mathrm{II}}} f\left(\bar{v}_{\mathrm{I}}, \bar{u}\right) f\left(\bar{v}_{\mathrm{II}}, \bar{v}_{\mathrm{I}}\right) .
\end{aligned}
$$

Here the sum is taken over all partitions $\bar{v} \Rightarrow\left\{\bar{v}_{\mathrm{I}}, \bar{v}_{\mathrm{II}}\right\}$.

$$
\begin{aligned}
& K_{n, m}^{(z)}(\bar{u} \mid \bar{v})=(1-z)^{m-n} \sum_{\bar{u} \Rightarrow\left\{\bar{u}_{\mathrm{I}}, \bar{u}_{\mathrm{II}}\right\}}(-z)^{\# \bar{u}_{\mathrm{I}}} f\left(\bar{u}_{\mathrm{II}}, \bar{v}\right) f\left(\bar{u}_{\mathrm{I}}, \bar{u}_{\mathrm{II}}\right), \\
& \bar{K}_{n, m}^{(z)}(\bar{u} \mid \bar{v})=(1-z)^{m-n} \sum_{\bar{u} \Rightarrow\left\{\bar{u}_{\mathrm{I}}, \bar{u}_{\mathbb{I}}\right\}}(-z)^{\# \bar{u}_{\mathrm{I}}} f\left(\bar{v}, \bar{u}_{\mathrm{II}}\right) f\left(\bar{u}_{\mathrm{II}}, \bar{u}_{\mathrm{I}}\right) .
\end{aligned}
$$

Here the sum is taken over all partitions $\bar{u} \Rightarrow\left\{\bar{u}_{\mathrm{I}}, \bar{u}_{\mathrm{II}}\right\}$.

Proof. Expanding the determinant (3.5) over diagonal minors we find

$$
\begin{aligned}
& \quad \operatorname{det}_{n}\left(\frac{f\left(v_{j}, \bar{v}_{j}\right) f\left(\bar{u}, v_{j}\right)}{h\left(v_{j}, v_{k}\right)}-z \delta_{j k}\right) \\
& =(-z)^{n}+\sum_{s=1}^{n}(-z)^{n-s} \sum_{1 \leq j_{1}<\cdots<j_{s} \leq n}\left(\prod_{p=1}^{s} f\left(v_{j_{p}}, \bar{v}_{j_{p}}\right) f\left(\bar{u}, v_{j_{p}}\right)\right) \operatorname{det} \frac{1}{h\left(v_{j_{i}}, v_{j_{k}}\right)} .
\end{aligned}
$$

The determinant in the right hand side is the Cauchy determinant, hence,

$$
\operatorname{det}_{s} \frac{1}{h\left(v_{j_{i}}, v_{j_{k}}\right)}=\prod_{\substack{p, q=1 \\ p \neq q}}^{s} \frac{1}{f\left(v_{j_{p}}, v_{j_{q}}\right)} .
$$

Thus, we obtain

$$
\begin{aligned}
& \operatorname{det}_{n}\left(\frac{f\left(v_{j}, \bar{v}_{j}\right) f\left(\bar{u}, v_{j}\right)}{h\left(v_{j}, v_{k}\right)}-z \delta_{j k}\right) \\
& =(-z)^{n}+\sum_{s=1}^{n}(-z)^{n-s} \sum_{1 \leq j_{1}<\cdots<j_{s} \leq n}\left(\prod_{p=1}^{s} f\left(v_{j_{p}}, \bar{v}_{j_{p}}\right) f\left(\bar{u}, v_{j_{p}}\right)\right) \prod_{\substack{p, q=1 \\
p \neq q}}^{s} \frac{1}{f\left(v_{j_{p}}, v_{j_{q}}\right)} .
\end{aligned}
$$

This is exactly the sum over partitions given by the first equation (A.8). The second equation (A.8) then follows by means of the replacement $c \rightarrow-c$. Equations (A.9) can be proved exactly in the same manner starting from the representation (3.6).

Proposition A.5.

$$
\bar{K}_{n, m}^{(z)}(\bar{u} \mid \bar{v})=(1-z)^{m-n} K_{m, n}^{(z)}(\bar{v} \mid \bar{u})
$$


Proof. Replacing $\bar{u} \leftrightarrow \bar{v}$ and $n \leftrightarrow m$ in (A.9) we obtain

$$
(1-z)^{m-n} K_{m, n}^{(z)}(\bar{v} \mid \bar{u})=\sum_{\bar{v} \Rightarrow\left\{\bar{v}_{\mathrm{I}}, \bar{v}_{\mathrm{II}}\right\}}(-z)^{\# \bar{v}_{\mathrm{I}}} f\left(\bar{v}_{\mathrm{II}}, \bar{u}\right) f\left(\bar{v}_{\mathrm{I}}, \bar{v}_{\mathrm{II}}\right) .
$$

Comparing this expansion with the second equation (A.8) we see that they coincide up to the labels of the subsets.

\section{Proposition A.6.}

$$
\begin{aligned}
K_{n, m}^{(z)}(\bar{u} \mid \bar{v}+c) & =\frac{(-z)^{n}(1-z)^{m-n}}{f(\bar{v}, \bar{u})} K_{m, n}^{(1 / z)}(\bar{v} \mid \bar{u}) . \\
\bar{K}_{n, m}^{(z)}(\bar{u} \mid \bar{v}-c) & =\frac{(-z)^{n}(1-z)^{m-n}}{f(\bar{u}, \bar{v})} \bar{K}_{m, n}^{(1 / z)}(\bar{v} \mid \bar{u}) .
\end{aligned}
$$

Proof. Using (3.5) we obtain

$$
K_{n, m}^{(z)}(\bar{u} \mid \bar{v}+c)=\operatorname{det}_{m}\left(-z \delta_{j k}+\frac{f\left(v_{j}, \bar{v}_{j}\right)}{f\left(v_{j}, \bar{u}\right) h\left(v_{j}, v_{k}\right)}\right)=\frac{(-z)^{m}}{f(\bar{v}, \bar{u})} \operatorname{det}\left(\delta_{j k} f\left(v_{j}, \bar{u}\right)-\frac{1}{z} \frac{f\left(v_{j}, \bar{v}_{j}\right)}{h\left(v_{j}, v_{k}\right)}\right) .
$$

On the other hand, using (3.6) for $K_{m, n}^{(1 / z)}(\bar{v} \mid \bar{u})$ we obtain

$$
K_{m, n}^{(1 / z)}(\bar{u} \mid \bar{v})=\left(1-\frac{1}{z}\right)^{n-m} \operatorname{det}_{m}\left(\delta_{j k} f\left(v_{j}, \bar{u}\right)-\frac{1}{z} \frac{f\left(v_{j}, \bar{v}_{j}\right)}{h\left(v_{j}, v_{k}\right)}\right) .
$$

Comparing (A.17) and (A.18) we arrive at (A.15). Equation (A.16) follows from the replacement $c \rightarrow-c$.

Proposition A.7. The function $K_{n, m}^{(z)}(\bar{u} \mid \bar{v})$ has poles at $u_{j}=v_{k}$. The residue at $u_{n}=v_{m}$ is given by

$$
\begin{aligned}
& \left.K_{n, m}^{(z)}(\bar{u} \mid \bar{v})\right|_{u_{n} \rightarrow v_{m}}=g\left(u_{n}, v_{m}\right) f\left(\bar{u}_{n}, u_{n}\right) f\left(v_{m}, \bar{v}_{m}\right) K_{n-1, m-1}^{(z)}\left(\bar{u}_{n} \mid \bar{v}_{m}\right)+r e g, \\
& \left.\bar{K}_{n, m}^{(z)}(\bar{u} \mid \bar{v})\right|_{u_{n} \rightarrow v_{m}}=g\left(v_{m}, u_{n}\right) f\left(u_{n}, \bar{u}_{n}\right) f\left(\bar{v}_{m}, v_{m}\right) \bar{K}_{n-1, m-1}^{(z)}\left(\bar{u}_{n} \mid \bar{v}_{m}\right)+r e g,
\end{aligned}
$$

where reg means regular part.

Proof. It is clear that the two equations (A.19) are related by the replacement $c \rightarrow-c$. To prove the first equation we use (3.6). Then for $u_{n}=v_{m}$ the pole occurs only in the matrix element $\delta_{n k} f\left(u_{n}, \bar{v}\right)$. The determinant reduces to the product of this element and the corresponding minor:

$$
\begin{aligned}
\left.K_{n, m}^{(z)}(\bar{u} \mid \bar{v})\right|_{u_{n} \rightarrow v_{m}}=(1-z)^{m-n} g\left(u_{n}, v_{m}\right) f\left(v_{m}, \bar{v}_{m}\right) & \\
& \quad \times \operatorname{det}_{n-1}\left(\delta_{j k} f\left(u_{j}, \bar{v}_{m}\right) f\left(u_{j}, v_{m}\right)-z \frac{f\left(u_{j}, \bar{u}_{j, n}\right) f\left(u_{j}, u_{n}\right)}{h\left(u_{j}, u_{k}\right)}\right)+r e g
\end{aligned}
$$


where $\bar{u}_{j, n}=\bar{u} \backslash\left\{u_{j}, u_{n}\right\}$. We see that for $u_{n}=v_{m}$ we can extract the factor $f\left(u_{j}, u_{n}\right)$ form the $j$-th row of the matrix. Thus,

$$
\begin{aligned}
\left.K_{n, m}^{(z)}(\bar{u} \mid \bar{v})\right|_{u_{n} \rightarrow v_{m}}=(1-z)^{m-n} g\left(u_{n}, v_{m}\right) f\left(v_{m}, \bar{v}_{m}\right) f\left(\bar{u}_{n}, u_{n}\right) & \\
& \times \operatorname{det}_{n-1}\left(\delta_{j k} f\left(u_{j}, \bar{v}_{m}\right)-z \frac{f\left(u_{j}, \bar{u}_{j, n}\right)}{h\left(u_{j}, u_{k}\right)}\right)+r e g,
\end{aligned}
$$

which ends the proof.

\section{A.2 Summation formulas}

Proposition A.8. Let $\bar{\xi}, \bar{u}$, and $\bar{v}$ be sets of arbitrary complex numbers such that $\# \bar{\xi}=l, \# \bar{u}=n$, and $\# \bar{v}=m$. Then

$$
\begin{gathered}
\sum_{\bar{\xi} \Rightarrow\left\{\bar{\xi}_{\mathrm{I}}, \bar{\xi}_{\mathrm{\xi}}\right\}} z_{2}^{l_{\mathrm{I}}} K_{n, l_{\mathrm{I}}}^{\left(z_{1}\right)}\left(\bar{u} \mid \bar{\xi}_{\mathrm{I}}\right) K_{m, l_{\mathrm{II}}}^{\left(z_{2}\right)}\left(\bar{v} \mid \bar{\xi}_{\mathrm{II}}\right) f\left(\bar{\xi}_{\mathrm{II}}, \bar{\xi}_{\mathrm{I}}\right) f\left(\bar{u}, \bar{\xi}_{\mathrm{I}}\right)=K_{n+m, l}^{\left(z_{1} z_{2}\right)}(\{\bar{u}, \bar{v}\} \mid \bar{\xi}), \\
\sum_{\bar{\xi} \Rightarrow\left\{\bar{\xi}_{\mathrm{I}}, \bar{\xi}_{\mathrm{II}}\right\}} z_{2}^{l_{\mathrm{l}}} \bar{K}_{n, l_{\mathrm{I}}}^{\left(z_{1}\right)}\left(\bar{u} \mid \bar{\xi}_{\mathrm{I}}\right) \bar{K}_{m, l_{\mathrm{II}}}^{\left(z_{2}\right)}\left(\bar{v} \mid \bar{\xi}_{\mathrm{II}}\right) f\left(\bar{\xi}_{\mathrm{I}}, \bar{\xi}_{\mathrm{I}}\right) f\left(\bar{\xi}_{\mathrm{I}}, \bar{u}\right)=\bar{K}_{n+m, l}^{\left(z_{1} z_{2}\right)}(\{\bar{u}, \bar{v}\} \mid \bar{\xi}) .
\end{gathered}
$$

Here $l_{\mathrm{I}}=\# \bar{\xi}_{\mathrm{I}}$ and $l_{\mathrm{II}}=\# \bar{\xi}_{\mathrm{I}}$. The sums are taken with respect to all partitions $\bar{\xi} \Rightarrow\left\{\bar{\xi}_{\mathrm{I}}, \bar{\xi}_{\mathrm{II}}\right\}$. There is no restriction on the cardinalities of the subsets.

Proof. It is clear that the two equations (A.22) are related by the replacement $c \rightarrow-c$. To prove the first equation we use (A.8):

$$
\begin{aligned}
& K_{n, l_{\mathrm{I}}}^{\left(z_{1}\right)}\left(\bar{u} \mid \bar{\xi}_{\mathrm{I}}\right)=\sum_{\bar{\xi}_{\mathrm{I}} \Rightarrow\left\{\bar{\xi}_{1}, \bar{\xi}_{2}\right\}}\left(-z_{1}\right)^{l_{2}} f\left(\bar{u}, \bar{\xi}_{1}\right) f\left(\bar{\xi}_{1}, \bar{\xi}_{2}\right), \\
& K_{m, l_{\mathrm{II}}}^{\left(z_{2}\right)}\left(\bar{v} \mid \bar{\xi}_{\mathrm{II}}\right)=\sum_{\bar{\xi}_{\mathrm{I}} \Rightarrow\left\{\bar{\xi}_{3}, \bar{\xi}_{4}\right\}}\left(-z_{2}\right)^{l_{4}} f\left(\bar{v}, \bar{\xi}_{3}\right) f\left(\bar{\xi}_{3}, \bar{\xi}_{4}\right) .
\end{aligned}
$$

Here we use Arabic numbers for numeration the subsets. The corresponding cardinalities are $l_{i}=\# \bar{\xi}_{i}, i=1,2,3,4$. Thus, $l_{\mathrm{I}}=l_{1}+l_{2}$ and $l_{\mathrm{II}}=l_{3}+l_{4}$. Denoting the left hand side of the first equation (A.22) by $\Lambda$ we obtain

$$
\begin{array}{rl}
\Lambda=\sum_{\bar{\xi} \Rightarrow\left\{\bar{\xi}_{1}, \bar{\xi}_{2}, \bar{\xi}_{3}, \bar{\xi}_{4}\right\}}(-1)^{l_{4}} z_{2}^{l_{1}+l_{2}+l_{4}}\left(-z_{1}\right)^{l_{2}} & f\left(\bar{u}, \bar{\xi}_{1}\right) f\left(\bar{v}, \bar{\xi}_{3}\right) f\left(\bar{\xi}_{1}, \bar{\xi}_{2}\right) f\left(\bar{\xi}_{3}, \bar{\xi}_{4}\right) \\
& \times f\left(\bar{u}, \bar{\xi}_{3}\right) f\left(\bar{u}, \bar{\xi}_{4}\right) f\left(\bar{\xi}_{3}, \bar{\xi}_{1}\right) f\left(\bar{\xi}_{3}, \bar{\xi}_{2}\right) f\left(\bar{\xi}_{4}, \bar{\xi}_{1}\right) f\left(\bar{\xi}_{4}, \bar{\xi}_{2}\right) .
\end{array}
$$

Setting $\left\{\bar{\xi}_{4}, \bar{\xi}_{1}\right\}=\bar{\xi}_{0}$ we find

$$
\begin{aligned}
\Lambda=f(\bar{u}, \bar{\xi}) \sum_{\bar{\xi} \Rightarrow\left\{\bar{\xi}_{0}, \bar{\xi}_{2}, \bar{\xi}_{3}\right\}} z_{2}^{l_{0}+l_{2}}\left(-z_{1}\right)^{l_{2}} \frac{f\left(\bar{v}, \bar{\xi}_{3}\right)}{f\left(\bar{u}, \bar{\xi}_{2}\right)} f\left(\bar{\xi}_{0}, \bar{\xi}_{2}\right) f\left(\bar{\xi}_{3}, \bar{\xi}_{0}\right) f\left(\bar{\xi}_{3}, \bar{\xi}_{2}\right) \\
\times \sum_{\bar{\xi}_{0} \Rightarrow\left\{\bar{\xi}_{1}, \bar{\xi}_{4}\right\}}(-1)^{l_{4}} f\left(\bar{\xi}_{4}, \bar{\xi}_{1}\right) .
\end{aligned}
$$


It was proved in 35] that for any set of variables $\bar{x}$ such that $\# \bar{x}=p$ the following identity holds:

$$
\sum_{\substack{\bar{x} \Rightarrow\left\{\bar{x}_{\mathrm{I}}, \bar{x}_{\mathrm{II}}\right\} \\
\# \bar{x}_{\mathrm{I}}=k}} f\left(\bar{x}_{\mathrm{II}}, \bar{x}_{\mathrm{I}}\right)=\sum_{\substack{\bar{x} \Rightarrow\left\{\bar{x}_{\mathrm{I}}, \bar{x}_{\mathrm{II}}\right\} \\
\# \bar{x}_{\mathrm{I}}=k}} f\left(\bar{x}_{\mathrm{I}}, \bar{x}_{\mathrm{II}}\right)=\left(\begin{array}{l}
p \\
k
\end{array}\right) .
$$

Here the sum is taken over partitions $\bar{x} \Rightarrow\left\{\bar{x}_{\mathrm{I}}, \bar{x}_{\mathrm{I}}\right\}$ such that the cardinality of the subset $\bar{x}_{\mathrm{I}}$ is fixed by $\# \bar{x}_{\mathrm{I}}=k, k \leq p$. Applying this result to the sum over partitions $\bar{\xi}_{0} \Rightarrow\left\{\bar{\xi}_{1}, \bar{\xi}_{4}\right\}$ we see that this sum vanishes if $\bar{\xi}_{0} \neq \emptyset$ :

$$
\sum_{\bar{\xi}_{0} \Rightarrow\left\{\bar{\xi}_{1}, \bar{\xi}_{4}\right\}}(-1)^{l_{4}} f\left(\bar{\xi}_{4}, \bar{\xi}_{1}\right)=\sum_{l_{4}=0}^{l_{0}}(-1)^{l_{4}}\left(\begin{array}{l}
l_{0} \\
l_{4}
\end{array}\right)=(1-1)^{l_{0}} .
$$

Thus, we obtain

$$
\Lambda=\sum_{\bar{\xi} \Rightarrow\left\{\bar{\xi}_{2}, \bar{\xi}_{3}\right\}}\left(-z_{1} z_{2}\right)^{l_{2}} f\left(\bar{v}, \bar{\xi}_{3}\right) f\left(\bar{u}, \bar{\xi}_{3}\right) f\left(\bar{\xi}_{3}, \bar{\xi}_{2}\right)=K_{n+m, l}^{\left(z_{1} z_{2}\right)}(\{\bar{u}, \bar{v}\} \mid \bar{\xi}),
$$

due to (A.8).

Replacing $\bar{\xi}$ by $\bar{\xi} \pm c$ and setting $z_{1}=z_{2}=1$ in (A.22) we obtain

$$
\begin{gathered}
\sum_{\bar{\xi} \Leftrightarrow\left\{\bar{\xi}_{\mathrm{I}}, \bar{\xi}_{\mathrm{II}}\right\}} K_{n, l_{\mathrm{I}}}^{(1)}\left(\bar{u}_{\mid} \mid \bar{\xi}_{\mathrm{I}}+c\right) K_{m, l_{\mathrm{II}}}^{(1)}\left(\bar{v} \mid \bar{\xi}_{\mathrm{II}}+c\right) \frac{f\left(\bar{\xi}_{\mathrm{II}}, \bar{\xi}_{\mathrm{I}}\right)}{f\left(\bar{\xi}_{\mathrm{II}}, \bar{u}\right)}=K_{n+m, k}^{(1)}(\{\bar{u}, \bar{v}\} \mid \bar{\xi}+c), \\
\sum_{\bar{\xi} \Rightarrow\left\{\bar{\xi}_{\mathrm{I}}, \bar{\xi}_{\mathrm{II}}\right\}} \bar{K}_{n, l_{\mathrm{I}}}^{(1)}\left(\bar{u} \mid \bar{\xi}_{\mathrm{I}}-c\right) \bar{K}_{m, l_{\mathrm{II}}}^{(1)}\left(\bar{v}^{\prime} \mid \bar{\xi}_{\mathrm{II}}-c\right) \frac{f\left(\bar{\xi}_{\mathrm{I}}, \bar{\xi}_{\mathrm{II}}\right)}{f\left(\bar{u}, \bar{\xi}_{\mathrm{II}}\right)}=\bar{K}_{n+m, k}^{(1)}(\{\bar{u}, \bar{v}\} \mid \bar{\xi}-c) .
\end{gathered}
$$

These formulas were used in sections 5.1 and 5.2 .

Proposition A.9. Let $\bar{u}$ and $\bar{v}$ be sets of arbitrary complex numbers such that $\# \bar{u}=n$ and $\# \bar{v}=m$. Then

$$
\begin{gathered}
\sum_{\bar{v} \Rightarrow\left\{\bar{v}_{\mathrm{I}}, \bar{v}_{\Pi}\right\}} z_{1}^{l_{\mathrm{II}}} K_{n, l_{\mathrm{I}}}^{\left(z_{2}\right)}\left(\bar{u} \mid \bar{v}_{\mathrm{I}}\right) f\left(\bar{v}_{\mathrm{I}}, \bar{v}_{\mathrm{II}}\right)=K_{n, m}^{\left(z_{2}-z_{1}\right)}(\bar{u} \mid \bar{v}), \\
\sum_{\bar{v} \Rightarrow\left\{\bar{v}_{\mathrm{I}}, \bar{v}_{\mathrm{II}}\right\}} z_{1}^{l_{\mathrm{II}}} \bar{K}_{n, l_{\mathrm{I}}}^{\left(z_{2}\right)}\left(\bar{u} \mid \bar{v}_{\mathrm{I}}\right) f\left(\bar{v}_{\mathrm{II}}, \bar{v}_{\mathrm{I}}\right)=\bar{K}_{n, m}^{\left(z_{2}-z_{1}\right)}(\bar{u} \mid \bar{v}) .
\end{gathered}
$$

Here $l_{\mathrm{II}}=\# \bar{v}_{\mathrm{II}}$. The sums are taken with respect to all partitions $\bar{v} \Rightarrow\left\{\bar{v}_{\mathrm{I}}, \bar{v}_{\mathrm{II}}\right\}$. There is no restriction on the cardinalities of the subsets.

Proof. Obviously, the two equations (A.30) are related by the replacement $c \rightarrow-c$, therefore, we prove only the first equation. Let

$$
\mathcal{L}=\sum_{\bar{v} \Rightarrow\left\{\bar{v}_{\mathrm{I}}, \bar{v}_{\mathrm{II}}\right\}} z_{1}^{l_{\mathrm{II}}} K_{n, l_{\mathrm{I}}}^{\left(z_{2}\right)}\left(\bar{u} \mid \bar{v}_{\mathrm{I}}\right) f\left(\bar{v}_{\mathrm{I}}, \bar{v}_{\mathrm{II}}\right)
$$


Using (A.8) we obtain

$$
\mathcal{L}=\sum_{\bar{v} \Rightarrow\left\{\bar{v}_{\mathrm{i}}, \bar{v}_{\mathrm{ii}}, \bar{v}_{\mathrm{II}}\right\}} z_{1}^{l_{\mathrm{II}}}\left(-z_{2}\right)^{l_{\mathrm{ii}}} f\left(\bar{u}, \bar{v}_{\mathrm{i}}\right) f\left(\bar{v}_{\mathrm{i}}, \bar{v}_{\mathrm{ii}}\right) f\left(\bar{v}_{\mathrm{i}}, \bar{v}_{\text {II }}\right) f\left(\bar{v}_{\mathrm{ii}}, \bar{v}_{\mathrm{II}}\right) .
$$

Here $l_{\mathrm{II}}=\# \bar{v}_{\mathrm{II}}, l_{\mathrm{ii}}=\# \bar{v}_{\mathrm{ii}}$, and the sum is taken with respect to all partitions $\bar{v} \Rightarrow\left\{\bar{v}_{\mathrm{i}}, \bar{v}_{\mathrm{ii}}, \bar{v}_{\mathrm{II}}\right\}$. Setting $\bar{v}_{0}=\left\{\bar{v}_{\mathrm{ii}}, \bar{v}_{\mathrm{II}}\right\}$ and $l_{0}=\# \bar{v}_{0}$ we find

$$
\mathcal{L}=\sum_{\bar{v} \Rightarrow\left\{\bar{v}_{\mathrm{i}}, \bar{v}_{0}\right\}} z_{1}^{l_{0}} f\left(\bar{u}, \bar{v}_{\mathrm{i}}\right) f\left(\bar{v}_{\mathrm{i}}, \bar{v}_{0}\right) \sum_{\bar{v}_{0} \Rightarrow\left\{\bar{v}_{\mathrm{ii}}, \overline{\mathrm{I}}_{\mathrm{II}}\right\}}\left(-\frac{z_{2}}{z_{1}}\right)^{l_{\mathrm{ii}}} f\left(\bar{v}_{\mathrm{ii}}, \bar{v}_{\mathrm{II}}\right) .
$$

Here we first have the sum over partitions $\bar{v} \Rightarrow\left\{\bar{v}_{\mathrm{i}}, \bar{v}_{0}\right\}$ and then the subset $\bar{v}_{0}$ is divided once more as $\bar{v}_{0} \Rightarrow\left\{\bar{v}_{\mathrm{ii}}, \bar{v}_{\mathrm{II}}\right\}$. Using (A.26) we find

$$
\sum_{\bar{v}_{0} \Rightarrow\left\{\bar{v}_{\mathrm{ii}}, \bar{v}_{\mathrm{II}}\right\}}\left(-\frac{z_{2}}{z_{1}}\right)^{l_{\mathrm{ii}}} f\left(\bar{v}_{\mathrm{ii}}, \bar{v}_{\mathrm{II}}\right)=\sum_{l_{\mathrm{ii}}=0}^{l_{0}}\left(-\frac{z_{2}}{z_{1}}\right)^{l_{\mathrm{ii}}}\left(\begin{array}{c}
l_{0} \\
l_{\mathrm{ii}}
\end{array}\right)=\left(1-\frac{z_{2}}{z_{1}}\right)^{l_{0}} .
$$

Substituting this result into A.33) we immediately arrive at

$$
\mathcal{L}=\sum_{\bar{v} \Rightarrow\left\{\bar{v}_{\mathrm{i}}, \bar{v}_{0}\right\}}\left(z_{1}-z_{2}\right)^{l_{0}} f\left(\bar{u}, \bar{v}_{\mathrm{i}}\right) f\left(\bar{v}_{\mathrm{i}}, \bar{v}_{0}\right)=K_{n, m}^{\left(z_{2}-z_{1}\right)}(\bar{u} \mid \bar{v}),
$$

due to (A.8).

\section{B Commutation relations of $t_{i j}(u)$ and $\nu_{i j}(u)$}

The RTT relation (2.5) yields to the following commutation relations:

$$
\left[t_{i j}(u), t_{k l}(v)\right]=g(u, v)\left(t_{k j}(v) t_{i l}(u)-t_{k j}(u) t_{i l}(v)\right) .
$$

In particular,

$$
\begin{aligned}
& t_{i j}(u) t_{i j}(v)=t_{i j}(v) t_{i j}(u), \quad \forall i, j, \\
& t_{11}(u) t_{12}(v)=f(v, u) t_{12}(v) t_{11}(u)+g(u, v) t_{12}(u) t_{11}(v), \\
& t_{22}(u) t_{12}(v)=f(u, v) t_{12}(v) t_{22}(u)+g(v, u) t_{12}(u) t_{22}(v), \\
& {\left[t_{21}(u), t_{12}(v)\right]=g(u, v)\left(t_{11}(v) t_{22}(u)-t_{11}(u) t_{22}(v)\right) .}
\end{aligned}
$$

In turn, commutation relations (B.3) and (B.4) imply the following multiple commutation relations [31]:

$$
\begin{aligned}
& t_{11}(\bar{u}) t_{12}(\bar{v})=(-1)^{n} \sum_{\# \bar{w}_{\mathrm{I}}=n} \bar{K}_{n}\left(\bar{u} \mid \bar{w}_{\mathrm{I}}+c\right) f\left(\bar{w}_{\mathrm{II}}, \bar{w}_{\mathrm{I}}\right) t_{12}\left(\bar{w}_{\mathrm{II}}\right) t_{11}\left(\bar{w}_{\mathrm{I}}\right), \\
& t_{22}(\bar{u}) t_{12}(\bar{v})=(-1)^{n} \sum_{\# \bar{w}_{\mathrm{I}}=n} K_{n}\left(\bar{u} \mid \bar{w}_{\mathrm{I}}+c\right) f\left(\bar{w}_{\mathrm{I}}, \bar{w}_{\mathrm{II}}\right) t_{12}\left(\bar{w}_{\mathrm{II}}\right) t_{22}\left(\bar{w}_{\mathrm{I}}\right) .
\end{aligned}
$$

Here $\# \bar{u}=n, \# \bar{v}=m, \bar{w}=\{\bar{u}, \bar{v}\}$, and $K_{n}$ is the Izergin determinant. The sums are taken over partitions $\bar{w} \Rightarrow\left\{\bar{w}_{\mathrm{I}}, \bar{w}_{\mathrm{II}}\right\}$ such that $\# \bar{w}_{\mathrm{I}}=n$.

The same commutation relations are valid for the modified operators $\nu_{i j}(u)$. 


\section{Symmetries of the Yangian}

Consider a mapping

$$
\phi(T(u))=T^{\tau}(-u),
$$

where $\tau$ is the diagonal transposition $A^{\tau}=\sigma^{1} A^{t} \sigma^{1}$ and $\sigma^{1}=\left(\begin{array}{ll}0 & 1 \\ 1 & 0\end{array}\right)$. It defines an automorphism of the Yangian of $\mathfrak{g l}_{2}$ [44]. This automorphism allows us to find the action of $\nu_{22}(u)$ on the modified Bethe vector knowing those for $\nu_{11}(u)$

$$
\phi\left(\nu_{11}(u) \nu_{12}(\bar{v})|0\rangle\right)=\nu_{22}(-u) \nu_{12}(-\bar{v})|0\rangle .
$$

Here we have to apply the following prescriptions: $\phi\left(\lambda_{i}(u)\right)=\lambda_{3-i}(-u)$ and $\phi\left(\beta_{i}\right)=\beta_{3-i}$.

Let us consider as an example the action (5.7). Applying the mapping $\phi$ to this equation we obtain

$$
\nu_{22}(-\bar{u}) \nu_{12}(-\bar{v})|0\rangle=\beta_{1}^{n} \sum_{\bar{w} \Rightarrow\left\{\bar{w}_{\mathrm{I}}, \bar{w}_{\text {II }}\right\}}\left(-\beta_{1}\right)^{-l} \lambda_{2}\left(-\bar{w}_{\mathrm{I}}\right) \bar{K}_{n, l}^{(1)}\left(\bar{u} \mid \bar{w}_{\mathrm{I}}-c\right) f\left(\bar{w}_{\mathrm{II}}, \bar{w}_{\mathrm{I}}\right) \nu_{12}\left(-\bar{w}_{\mathrm{II}}\right)|0\rangle .
$$

Changing $\bar{u} \rightarrow-\bar{u}$ and $\bar{v} \rightarrow-\bar{v}$ we arrive at

$$
\nu_{22}(\bar{u}) \nu_{12}(\bar{v})|0\rangle=\beta_{1}^{n} \sum_{\bar{w} \Rightarrow\left\{\bar{w}_{\mathrm{I}}, \bar{w}_{\mathrm{II}}\right\}}\left(-\beta_{1}\right)^{-l} \lambda_{2}\left(\bar{w}_{\mathrm{I}}\right) \bar{K}_{n, l}^{(1)}\left(-\bar{u} \mid-\bar{w}_{\mathrm{I}}-c\right) f\left(\bar{w}_{\mathrm{I}}, \bar{w}_{\mathrm{II}}\right) \nu_{12}\left(\bar{w}_{\mathrm{II}}\right)|0\rangle .
$$

Finally, using (A.2) we reproduce equation (5.8).

\section{References}

[1] S. Belliard, R. Pimenta, Slavnov and Gaudin formulas for models without U(1) symmetry: the twisted XXX chain, SIGMA 11 (2015) 099, arXiv:1506.06550.

[2] S. Belliard, N. A. Slavnov, B. Vallet, Modified algebraic Bethe Ansatz: twisted XXX case, to appear in SIGMA (2018), arXiv:1804.00597.

[3] V. E. Korepin, N. M. Bogoliubov and A. G. Izergin, Quantum Inverse Scattering Method and Correlation Functions, Cambridge: Cambridge Univ. Press, 1993.

[4] V. E. Korepin, Calculation of norms of Bethe wave functions, Comm. Math. Phys. 86 (1982) 391-418.

[5] N.A. Slavnov, Calculation of scalar products of wave functions and form factors in the framework of the algebraic Bethe ansatz, Theor. Math. Phys. 79 (1989) 502-508.

[6] N. Yu. Reshetikhin, Calculation of the norm of Bethe vectors in models with SU(3)-symmetry, Zap. Nauchn. Sem. LOMI 150 (1986) 196-213; J. Math. Sci. 46 (1989) 1694-1706 (Engl. transl.).

[7] S. Belliard, S. Pakuliak, E. Ragoucy and N. A. Slavnov, The algebraic Bethe ansatz for scalar products in SU(3)-invariant integrable models, J. Stat. Mech. (2012) P09003, arXiv:1207.0956 
[8] A. Hutsalyuk, A. Liashyk, S. Z. Pakuliak, E. Ragoucy, N. A. Slavnov, Scalar products of Bethe vectors in the models with $\mathfrak{g l}(m \mid n)$ symmetry, Nucl. Phys. B923 (2017) 277-311, arXiv: 1704.08173.

[9] N. A. Slavnov, Scalar products in GL(3)-based models with trigonometric R-matrix. Determinant representation, J. Stat. Mech. (2015) P03019, arXiv:1501.06253.

[10] N. Kitanine, J. M. Maillet, V. Terras, Form factors of the XXZ Heisenberg spin-12 finite chain, Nucl. Phys. B 554 (1999) 647-678, arXiv:math-ph/9807020.

[11] J. M. Maillet, V. Terras, On the quantum inverse scattering problem, Nucl. Phys. B 575 (2000) 627-644, hep-th/9911030.

[12] N. Kitanine, K. K. Kozlowski, J. M. Maillet, N. A. Slavnov, V. Terras, On the thermodynamic limit of form factors in the massless XXZ Heisenberg chain, J. Math. Phys., 50:9 (2009), 095209, arXiv:0903.2916.

[13] N. Kitanine, K. Kozlowski, J. M. Maillet, N. A. Slavnov, V. Terras, The thermodynamic limit of particle-hole form factors in the massless XXZ Heisenberg chain, J. Stat. Mech. Theory Exp., 2011, P05028.

[14] S. Belliard, S. Pakuliak, E. Ragoucy and N. A. Slavnov, Form factors in SU(3)-invariant integrable models, J. Stat. Mech. 1309 (2013) P04033, arXiv:1211.3968.

[15] S. Pakuliak, E. Ragoucy, N. A. Slavnov, Form factors in quantum integrable models with GL(3)-invariant R-matrix, Nucl. Phys. B 881 (2014) 343-368, arXiv:1312.1488.

[16] S. Pakuliak, E. Ragoucy, N. A. Slavnov, Form factors of local operators in a one-dimensional two-component Bose gas, J. Phys. A48 (2015) 435001, arXiv:1503.00546.

[17] A. Hutsalyuk, A. Liashyk, S. Z. Pakuliak, E. Ragoucy, N. A. Slavnov, Form factors of the monodromy matrix entries in $\mathfrak{g l}(2 \mid 1)$-invariant integrable models, Nucl. Phys. B911 (2016), 902-927, arXiv:1607.04978.

[18] J. Fuksa, N. Slavnov, Form factors of local operators in supersymmetric quantum integrable models, J. Stat. Mech. (2017) 043106, arXiv:1701.05866.

[19] M. Dugave, F. Göhmann, K. K. Kozlowski, J. Suzuki, Thermal form factors of the XXZ chain and the large-distance asymptotics of its temperature dependent correlation functions, J. Stat. Mech.: Theor. Exp. (2013) P07010, arXiv:1305.0118.

[20] B. Pozsgay, W.-V. van Gerven Oei and M. Kormos, On Form Factors in nested Bethe Ansatz systems, J. Phys. A: Math. Gen. 45 (2012) 465007, arXiv:1204.4037.

[21] N. Kitanine, K. K. Kozlowski, J. M. Maillet, N. A. Slavnov, V. Terras, A form factor approach to the asymptotic behavior of correlation functions, J. Stat. Mech. Theory Exp., 2011, P12010 , 28 pp., arXiv: 1110.0803. 
[22] N. Kitanine, K. K. Kozlowski, J. M. Maillet, N. A. Slavnov, V. Terras, Form factor approach to dynamical correlation functions in critical models, J. Stat. Mech. Theory Exp., 2012, P09001 , 33 pp., arXiv:1206.2630.

[23] N. Kitanine, K. K. Kozlowski, J. M. Maillet, V.Terras Long-distance asymptotic behaviour of multi-point correlation functions in massless quantum models, J. Stat. Mech.: Theory Exp., (2014) P05011, arXiv:1312.5089.

[24] M. Dugave, F. Göhmann, K. K. Kozlowski, J. Suzuki, Thermal form factor approach to the ground-state correlation functions of the XXZ chain in the antiferromagnetic massive regime, J. Phys. A 49 (2016) 394001, arXiv:1605.07968.

[25] F. Göhmann, M. Karbach, A. Klümper, K. K. Kozlowski, J. Suzuki, Thermal form-factor approach to dynamical correlation functions of integrable lattice models, J. Stat. Mech.: Theor. Exp. (2017) P113106, arXiv:1708.04062.

[26] J. S. Caux, J. M. Maillet, Computation of Dynamical Correlation Functions of Heisenberg Chains in a Magnetic Field, Phys. Rev. Lett. 95 (2005) 077201, arXiv:cond-mat/0502365.

[27] J.-S. Caux, P. Calabrese, N. A. Slavnov, One-particle dynamical correlations in the onedimensional Bose gas, J. Stat. Mech. 0701 (2007) P01008, arXiv:cond-mat/0611321.

[28] M. Panfil, J.-S. Caux, Finite temperature correlations in the Lieb-Liniger 1D Bose gas, Phys. Rev. A 89 (2014) 033605, arXiv:1308.2887.

[29] K. K. Kozlowski, E. Ragoucy, Asymptotic behaviour of two-point functions in multi-species models, Nucl. Phys. B 906 (2016) 241, arXiv:1601.04475

[30] N. Kitanine, J. M. Maillet, V. Terras, Correlation functions of the XXZ Heisenberg spin-1/2 chain in a magnetic field, Nucl.Phys. B 567 (2000) 554-582, arXiv:math-ph/9907019.

[31] S. Belliard, S. Pakuliak, E. Ragoucy and N. A. Slavnov, Bethe vectors of GL(3)-invariant integrable models, J. Stat. Mech.: Theory Exp. (2013) P02020, arXiv:1210.0768.

[32] A. Hutsalyuk, A. Liashyk, S. Pakuliak, E. Ragoucy, N.A. Slavnov, Multiple actions of the monodromy matrix in $\mathfrak{g l}(2 \mid 1)$-invariant integrable models, SIGMA 12 (2016) 099, arXiv: 1605.06419.

[33] N. A. Slavnov, Multiple commutation relations in the models with $\mathfrak{g l}(2 \mid 1)$ symmetry, Theor. Math. Phys., 189:2 (2016) 1624-1644, arXiv:1604.05343.

[34] N. Gromov, F. Levkovich-Maslyuk, G. Sizov, New Construction of Eigenstates and Separation of Variables for $S U(N)$ Quantum Spin Chains JHEP 09 (2017) 111, arXiv:1610.08032.

[35] S. Belliard, N. A. Slavnov, A note on $\mathfrak{g l}_{2}$-invariant Bethe vectors, JHEP 31 (2018), arXiv: 1802.07576.

[36] A. Liashyk and N. A. Slavnov, On Bethe vectors in $\mathfrak{g l}_{3}$-invariant integrable models, arXiv:1803.07628v1. 
[37] A. G. Izergin, Partition function of the six-vertex model in a finite volume, Dokl. Akad. Nauk SSSR 297 (1987) 331-333; Sov. Phys. Dokl. 32 (1987) 878-879 (Engl. transl.).

[38] A. Gorsky, A. Zabrodin, A. Zotov, Spectrum of quantum transfer matrices via classical manybody systems, JHEP (2014) 070, arXiv:1310.6958.

[39] O. Foda and M. Wheeler, Partial domain wall partition functions, J. of High Ener. Phys. 7 ,(2012) 186, arXiv:1205.4400

[40] L. D. Faddeev, E. K. Sklyanin and L. A. Takhtajan, Quantum Inverse Problem. I, Theor. Math. Phys. 40 (1979) 688-706.

[41] J. Cao, W. Yang, K. Shi and Y. Wang, Off-diagonal Bethe ansatz and exact solution a topological spin ring, Phys. Rev. Lett. 111 (2013) 137201, arXiv:1305.7328.

[42] J. Cao, W. Yang, K. Shi and Y. Wang, Off-diagonal Bethe ansatz for exactly solvable models, Springer, 2015.

[43] N. Kitanine, J.M. Maillet, G. Niccoli and V. Terras, On determinant representations of scalar products and form factors in the SoV approach: the XXX case, J. Phys. A, Vol. 49 (Special Issue) (2016) 104002, arXiv: 1506.02630

[44] A. Molev, Yangians and Classical Lie Algebras. Mathematical Surveys and Monographs, 143. American Mathematical Society, Providence, RI, 2007.

[45] X. Zhang, Y.-Y. Li, W. L. Yang, K. Shi and Y. Wang, Bethe states of the XXZ spin- $\frac{1}{2}$ chain with arbitrary boundary fields, Nucl. Phys. B 893 (2015), 70-88, arXiv:1412.6905

[46] S. Belliard and R. Pimenta, Modified algebraic Bethe ansatz for XXZ chain on the segment II -general cases, Nuclear Phys. B894 (2015) 527-552, arXiv:1412.7511. 